\title{
Measure of noncompactness for an infinite system of fractional Langevin equation in a sequence space
}

\author{
Ahmed Salem ${ }^{1 *}$ (D) Hashim M. Alshehri ${ }^{1}$ and Lamya Almaghamsi
}

\author{
"Correspondence: \\ ahmedsalem74@hotmail.com \\ 'Department of Mathematics, \\ Faculty of Science, King Abdulaziz \\ University, P.O. Box 80203, Jeddah, \\ 21589, Saudi Arabia \\ Full list of author information is \\ available at the end of the article
}

\begin{abstract}
A new sequence space related to the space $\ell_{p}, 1 \leq p<\infty$ (the space of all absolutely $p$-summable sequences) is established in the present paper. It turns out that it is Banach and a BK space with Schauder basis. The Hausdorff measure of noncompactness of this space is presented and proven. This formula with the aid the Darbo's fixed point theorem is used to investigate the existence results for an infinite system of Langevin equations involving generalized derivative of two distinct fractional orders with three-point boundary condition.
\end{abstract}

MSC: 34A08; 34A12; 47H08; 47H10; 46B45

Keywords: Infinite system; Fraction Langevin equation; Measure of noncompactness; Darbo's fixed point theorem; Sequence space

\section{Introduction}

Infinite systems of differential equations play a significant role in many subjects of nonlinear analysis. The infinite systems of ODEs represent some problems faced within the theories of neural nets, branching processes, and dissociation of polymers. Therefore, the notion of infinite systems of differential equations is considered a substantial part of the theory of differential equations, especially in Banach spaces $[1,2]$. To date, many existence results have been obtained for the infinite systems of ODEs in Banach spaces. Banas and Lecko [3] discussed the existence of solutions for an infinite system

$$
x_{i}^{\prime}=f_{i}\left(t, x_{0}, x_{1}, x_{2}, \ldots\right), \quad t \in[0, T]
$$

with the initial conditions $x_{i}(0)=x_{i}^{0}, i=\mathbb{N}_{0}$ in the classical Banach sequence spaces $c_{0}, c$, and $\ell_{1}$. In 2012, Mursaleen and Mohiuddineb [4] investigated it in the space $\ell_{p}, p>1$. Also, Mursaleen [5] investigated the same infinite system on the sequence space $n(\phi)$ of W.L.C. Sargent.

(c) The Author(s) 2021. This article is licensed under a Creative Commons Attribution 4.0 International License, which permits use, sharing, adaptation, distribution and reproduction in any medium or format, as long as you give appropriate credit to the original author(s) and the source, provide a link to the Creative Commons licence, and indicate if changes were made. The images or other third party material in this article are included in the article's Creative Commons licence, unless indicated otherwise in a credit line to the material. If material is not included in the article's Creative Commons licence and your intended use is not permitted by statutory regulation or exceeds the permitted use, you will need to obtain permission directly from the copyright holder. To view a copy of this licence, visit http://creativecommons.org/licenses/by/4.0/ 
The authors in $[6,7]$ considered the second-order infinite system

$$
x_{i}^{\prime \prime}=-f_{i}\left(t, x_{1}, x_{2}, x_{3}, \ldots\right), \quad t \in[0, T]
$$

with the initial conditions $x_{i}(0)=x_{i}(T), i \in \mathbb{N}$ in the space $\ell_{p}, p>1$.

Mursaleen [8] discussed the fractional infinite system

$$
D^{\alpha} u_{i}(t)=f_{i}(t, u(t)), \quad t \in(0, T), 1<\alpha<2,
$$

where $u(t)=\left\{u_{i}(t)\right\}_{i=0}^{\infty}$, with the initial conditions

$$
u_{i}(0)=u_{i}^{0}=0, \quad u_{i}(T)=a u_{i}(\xi), \quad i \in \mathbb{N}_{0}, \quad a \xi^{\alpha-1}<T^{\alpha-1}
$$

in Banach spaces, where $D^{\alpha}$ is the $\mathrm{R}-\mathrm{L}$ fractional derivative of order $\alpha$.

Recently, Seemab and Rehman [9] investigated the fractional infinite system

$$
{ }_{c}^{\rho} D^{\alpha} u_{i}(t)=f_{i}\left(t, u(t), \psi\left({ }_{c}^{\rho} D^{\beta} u(t)\right)\right), \quad t \in(0,1)
$$

in the Banach space $c_{0}$, where $u(t)=\left\{u_{i}(t)\right\}_{i=1}^{\infty}$ and $\psi: c_{0} \rightarrow c_{0}$ is a continuous linear function such that $\psi(0)=0$, with the initial conditions

$$
u_{i}(1)=a u_{i}(\xi), \quad u_{i}^{\prime}(0)=0, \quad i \in \mathbb{N}, \quad a \xi^{\alpha-1}<1,
$$

where $0<\rho \leq 1,1<\alpha \leq 2,0<\beta<1$, and ${ }_{c}^{\rho} D^{\alpha}$ is the Caputo generalized fractional derivative.

Fractional calculus, differentiation and integration, appears naturally in several fields of science and engineering; see, for instance, $[10]$ and more recent $[11,12]$, and the references given therein. A large number of existence results of differential and fractional differential equations have been formulated in terms of measures of noncompactness. Measures of noncompactness provide helpful information, which is extensively used in the theory of integral and integro-differential equations. Especially, the measure of noncompactness has been used extensively by many authors when studying infinite systems of differential and fractional differential equations.

Motivated by the former contributions, we consider the infinite system of the generalized Langevin equations

$$
{ }_{c}^{\rho_{i}} D^{\lambda_{i}}\left({ }_{c}^{\rho_{i}} D^{\mu_{i}}+\xi_{i}\right) u_{i}(t)=h_{i}\left(t, u(t), \phi\left({ }_{c}^{\rho} D^{v} u(t)\right)\right), \quad t \in[0,1], i \in \mathbb{N},
$$

where $u(t)=\left\{u_{i}(t)\right\}_{i=1}^{\infty} \in \ell_{p}, \phi\left({ }_{c}^{\rho} D^{v} u(t)\right)=\left\{\phi_{i}\left({ }_{c}^{\rho_{i}} D^{v_{i}} u_{i}(t)\right)\right\}_{n=1}^{\infty} \in \ell_{p}, \phi_{i}:[0,1] \times \ell_{p} \rightarrow \ell_{p}$ are continuous functions, $h_{i}:[0,1] \times \ell_{p} \times \ell_{p} \rightarrow \ell_{p}$ are continuously differentiable functions, $0<\rho_{i} \leq 1,1<\lambda_{i} \leq 2,0<v_{i}<\mu_{i} \leq 1,{ }_{c}^{\rho} D^{\alpha}$ is the generalized Liouville-Caputo derivative as in [13], and the space $\ell_{p}, 1 \leq p<\infty$ is the space of all absolutely $p$-summable sequences.

This infinite system is subject to the boundary conditions

$$
u_{i}(0)=0, \quad{ }_{c}^{\rho_{i}} D^{\mu_{i}} u_{i}(0)=0, \quad u_{i}(1)=a_{i} u_{i}\left(\eta_{i}\right)
$$

where $a_{i} \in \mathbb{R}, 0<\eta_{i}<1$ and $a_{i} \eta_{i}^{\mu_{i}-1} \neq 1$ for all $i \in \mathbb{N}$. 
By using the measure of noncompactness technique and applying the Darbo's fixed point theorem, we investigate the existence of solutions for the infinite system (1.1)-(1.2) in the Banach spaces $\ell_{p}, p \geq 1$.

It worth pointing out that the Langevin equations (formulated by Langevin in 1908) present an accurate way to describe the evolution of physical phenomena in a fluctuating environment [14]. There is a clear progress on fractional Langevin equations in physics [15-19]. New results on the existence of solutions for fractional Langevin equations under variety of boundary value conditions have been published; see [20-29] and the references mentioned therein.

\section{Preliminaries}

This section is divided into three subsections: The first introduces the concepts and the main results in fractional calculus that the paper needs. The second subsection represents a brief review on the measure of noncompactness and its applications when investigating the existence of solutions for differential equations. The last subsection presents a brief review about the sequence spaces used in this paper.

\subsection{Fractional calculus}

In our paper, we deal with the generalized Liouville-Caputo derivative which is considered a generalization for many known fractional derivatives [15]. Historically, in 2011, Katugampola [30] introduced a new version of fractional integral given by

$$
{ }^{\rho} I_{a}^{v} f(x)=\frac{\rho^{1-v}}{\Gamma(v)} \int_{a}^{x} s^{\rho-1}\left(x^{\rho}-s^{\rho}\right)^{v-1} f(s) d s
$$

where $\rho$ and $v$ are positive real numbers, while the function $f \in \mathbb{X}_{c}^{p}(a, b)$ (the space of Lebesgue measurable functions). He proved that this fractional operator satisfies the semigroup property:

$$
\begin{aligned}
& { }^{\rho} I_{a}^{v \rho} I_{a}^{\mu} f(x)={ }^{\rho} I_{a}^{\nu+\mu} f(x), \quad v>0, \mu>0, \\
& { }^{\rho} I_{a}^{v}\left(x^{\rho}-a^{\rho}\right)^{\delta}=\frac{\Gamma(\delta+1)}{\rho^{\nu} \Gamma(v+\delta+1)}\left(x^{\rho}-a^{\rho}\right)^{\delta+\nu}, \quad v>0, \delta>-1 .
\end{aligned}
$$

The importance of this approach comes from the fact that it is a generalization of Riemann-Liouville and Hadamard fractional integrals. It is easy to show that

$$
\begin{aligned}
& \lim _{\rho \rightarrow 1}{ }^{\rho} I_{a}^{v} f(x)=\frac{1}{\Gamma(v)} \int_{a}^{x}(x-s)^{v-1} f(s) d s={ }^{\mathrm{RL}} I_{a}^{v} f(x), \\
& \lim _{\rho \rightarrow 0}{ }^{\rho} I_{a}^{v} f(x)=\frac{1}{\Gamma(v)} \int_{a}^{x}\left(\log \frac{x}{s}\right)^{\nu-1} f(s) \frac{d s}{s}={ }^{\mathrm{H}} I_{a}^{v} f(x),
\end{aligned}
$$

where ${ }^{\mathrm{RL}} I_{a}^{v}$ and ${ }^{\mathrm{H}} I_{a}^{v}$ are the Riemann-Liouville and Hadamard fractional integral operators, respectively. Also, it is considered a special case of Erdélyi-Kober fractional integral operator [31, formula (1.1.17)]

$$
I_{\rho}^{\gamma, v} f(x)=\frac{\rho x^{-\rho(v+\gamma)}}{\Gamma(v)} \int_{a}^{x} s^{\rho(\gamma+1)-1}\left(x^{\rho}-s^{\rho}\right)^{\nu-1} f(s) d s, \quad v>0, \rho>0, \gamma \in \mathbb{R},
$$

as ${ }^{\rho} I_{a}^{v} f(x)=x^{\rho v} \rho^{-v} I_{\rho}^{0, v} f(x)$. 
Jarad et al. [32] introduced a new version of a fractional derivative in the Caputo sense which later became known as the generalized Liouville-Caputo derivative, given by

$$
{ }_{c}^{\rho} \mathcal{D}_{a}^{v} f(x)=\left({ }^{\rho} I_{a}^{n-v}\left(x^{1-\rho} \frac{d}{d x}\right)^{n} f\right)(x), \quad n-1<v \leq n, n \in \mathbb{N} .
$$

It is worth mentioning that the previous formula tends to Caputo derivative as $\rho \rightarrow 1$ and tends to Caputo-Hadamard derivative as $\rho \rightarrow 0$. Some of its semigroup properties are presented as follows.

Lemma 2.1 ([15,32]) Suppose that $n \in \mathbb{N}, n-1<v \leq n, 0<\rho \leq 1$, and $f \in \mathbb{X}_{c}^{p}(a, b)$. Then we have

- ${ }_{c}^{\rho} \mathcal{D}_{a}^{v} c=0$ where $c$ is a constant,

- ${ }_{c}^{\rho} \mathcal{D}_{a}^{v}\left(x^{\rho}-a^{\rho}\right)^{\delta}=\frac{\rho^{v} \Gamma(\delta+1)}{\Gamma(\delta-v+1)}\left(x^{\rho}-a^{\rho}\right)^{\delta-v}, \quad v>0, \delta>-1, \delta \neq 0,1, \ldots, n-1$,

- ${ }_{c}^{\rho} \mathcal{D}_{a}^{v \rho} I_{a}^{\alpha} f(x)={ }^{\rho} I_{a}^{\alpha-v} f(x), \quad \alpha \geq v$,

- ${ }^{\rho} I_{a c}^{v \rho} \mathcal{D}_{a}^{v} f(x)=f(x)-\sum_{r=0}^{n-1} c_{r}\left(x^{\rho}-a^{\rho}\right)^{r} \quad$ where $c_{r}, r=0,1, \ldots, n-1$ are constants.

From here onward, we replace ${ }^{\rho} I_{0}^{\lambda}$ and ${ }_{c}^{\rho} D_{0}^{\lambda}$ by ${ }^{\rho} I^{\lambda}$ and ${ }_{c}^{\rho} D^{\lambda}$, respectively.

Lemma 2.2 Suppose that the function $h:[0,1] \rightarrow \mathbb{R}$ is a continuous function, $\rho \in(0,1]$, $\lambda \in(1,2]$, and $\mu \in(0,1]$. Then, the linear boundary value problem

$$
{ }_{c}^{\rho} D^{\lambda}\left({ }_{c}^{\rho} D^{\mu}+\xi\right) u(t)=h(t), \quad t \in[0,1], \xi \in \mathbb{R},
$$

subject to the conditions

$$
u(0)=0, \quad{ }_{c}^{\rho} D^{\mu} u(0)=0, \quad u(1)=a u(\eta), \quad a \in \mathbb{R}, 0<\eta<1,
$$

has the unique solution

$$
u(t)=\int_{0}^{1} \mathcal{F}(t, s, \lambda, 0) h(s) d s-\xi \int_{0}^{1} \mathcal{F}(t, s, 0,0) u(s) d s,
$$

where

$$
\mathcal{F}(t, s, \lambda, v)=\frac{\rho^{1+\nu-\lambda-\mu} s^{\rho-1}}{\Gamma(\lambda+\mu-v)} \begin{cases}\mathcal{F}_{1}(t, s, \lambda, v), & 0 \leq t \leq \eta<1 \\ \mathcal{F}_{2}(t, s, \lambda, v), & 0<\eta \leq t \leq 1\end{cases}
$$

and $\mathcal{F}_{k}(t, s, \lambda, v), k=1,2$ are given by

$$
\mathcal{F}_{1}(t, s, \lambda, \nu)= \begin{cases}\left(t^{\rho}-s^{\rho}\right)^{\lambda+\mu-\nu-1}+\frac{t^{\rho(\mu-\nu+1)}}{\Omega}\left[a\left(\eta^{\rho}-s^{\rho}\right)^{\lambda+\mu-1}-\left(1-s^{\rho}\right)^{\lambda+\mu-1}\right], & 0 \leq s \leq t, \\ \frac{t^{\rho(\mu-v+1)}}{\Omega}\left[a\left(\eta^{\rho}-s^{\rho}\right)^{\lambda+\mu-1}-\left(1-s^{\rho}\right)^{\lambda+\mu-1}\right], & t \leq s \leq \eta, \\ -\frac{t^{\rho(\mu-v+1)}}{\Omega}\left(1-s^{\rho}\right)^{\lambda+\mu-1}, & \eta \leq s \leq 1,\end{cases}
$$




$$
\mathcal{F}_{2}(t, s, \lambda, \nu)= \begin{cases}\left(t^{\rho}-s^{\rho}\right)^{\lambda+\mu-\nu-1}+\frac{t^{\rho(\mu-v+1)}}{\Omega}\left[a\left(\eta^{\rho}-s^{\rho}\right)^{\lambda+\mu-1}-\left(1-s^{\rho}\right)^{\lambda+\mu-1}\right], & 0 \leq s \leq \eta, \\ \left(t^{\rho}-s^{\rho}\right)^{\lambda+\mu-\nu-1}-\frac{t^{\rho}(\mu-v+1)}{\Omega}\left(1-s^{\rho}\right)^{\lambda+\mu-1}, & \eta \leq s \leq t, \\ -\frac{t^{\rho}(\mu-v+1)}{\Omega}\left(1-s^{\rho}\right)^{\lambda+\mu-1}, & t \leq s \leq 1,\end{cases}
$$

where

$$
\Omega=\frac{\Gamma(\lambda+\mu-v) \Gamma(\mu+2)}{\Gamma(\mu-v+2) \Gamma(\lambda+\mu)}\left(1-a \eta^{\rho(\mu+1)}\right) \neq 0 .
$$

Proof Apply ${ }^{\rho} I^{\lambda}$ on both sides of (2.5) twice in succession using the semigroup property (2.2) and the relation (2.3) to obtain

$$
\begin{aligned}
& { }_{c}^{\rho} D^{\mu} u(t)+\xi u(t)={ }^{\rho} I^{\lambda} h(t)+c_{0}+c_{1} t^{\rho}, \\
& u(t)={ }^{\rho} I^{\lambda+\mu} h(t)-\xi^{\rho} I^{\mu} u(t)+c_{0} \frac{t^{\rho \mu}}{\rho^{\mu} \Gamma(\mu+1)}+c_{1} \frac{t^{\rho(\mu+1)}}{\rho^{\mu} \Gamma(\mu+2)}+c_{2} .
\end{aligned}
$$

Inserting the first and second conditions into (2.9) and (2.10) gives $c_{0}=c_{2}=0$. By applying the last condition, we find that

$$
\frac{c_{1} \Omega}{\rho^{\mu} \Gamma(\mu+2)}=a^{\rho} I^{\lambda+\mu} h(\eta)-{ }^{\rho} I^{\lambda+\mu} h(1)-a \xi^{\rho} I^{\mu} u(\eta)+\xi^{\rho} I^{\mu} u(1) .
$$

Substituting into (2.10) gives

$$
\begin{aligned}
u(t)= & \frac{\rho^{1-\lambda-\mu}}{\Gamma(\lambda+\mu)} \int_{0}^{t} \frac{s^{\rho-1} h(s)}{\left(t^{\rho}-s^{\rho}\right)^{1-\lambda-\mu}} d s-\frac{\xi \rho^{1-\mu}}{\Gamma(\mu)} \int_{0}^{t} \frac{s^{\rho-1} u(s)}{\left(t^{\rho}-s^{\rho}\right)^{1-\mu}} d s \\
& +\frac{t^{\rho(\mu+1)} \rho^{1-\mu}}{\Omega}\left(\frac{a \rho^{-\lambda}}{\Gamma(\lambda+\mu)} \int_{0}^{\eta} \frac{s^{\rho-1} h(s)}{\left(\eta^{\rho}-s^{\rho}\right)^{1-\lambda-\mu}} d s-\frac{a \xi}{\Gamma(\mu)} \int_{0}^{\eta} \frac{s^{\rho-1} u(s)}{\left(\eta^{\rho}-s^{\rho}\right)^{1-\mu}} d s\right. \\
& \left.-\frac{\rho^{-\lambda}}{\Gamma(\lambda+\mu)} \int_{0}^{1} \frac{s^{\rho-1} h(s)}{\left(1-s^{\rho}\right)^{1-\lambda-\mu}} d s+\frac{\xi}{\Gamma(\mu)} \int_{0}^{1} \frac{s^{\rho-1} u(s)}{\left(1-s^{\rho}\right)^{1-\mu}} d s\right),
\end{aligned}
$$

which gives the kernels in (2.7) and (2.8). Conversely, inserting (2.7) into the left-hand side of (2.5) and using the first three relations of Lemma 2.1, we obtain the right-hand side of (2.5). Also, it is not difficult to see that the solution (2.7) satisfies all conditions of (2.6). The proof is complete.

\subsection{Hausdorff measure of noncompactness}

There are many measures of noncompactness. The three main and most frequently used measures are Kuratowski, Istratescu, and Hausdorff measures of noncompactness [1]. Here, we deal with Hausdorff measure of noncompactness that needs the following notions and definitions.

Definition 2.1 Consider the Banach space $\left(\mathbb{E},\|\cdot\|_{\mathbb{E}}\right)$ and a nonempty and bounded subset $\mathscr{N}_{\mathbb{E}}$ of $\mathbb{E}$. Then the mapping $\beta: \mathscr{N}_{\mathbb{E}} \rightarrow[0, \infty)$ is called the Hausdorff measure of noncompactness of a set $\mathscr{N} \subset \mathscr{N}_{\mathbb{E}}$ and is defined as

$$
\beta(\mathscr{N})=\inf \{\epsilon>0 \mid \mathscr{N} \text { has a finite } \epsilon \text {-net in } \mathbb{E}\} .
$$


In order to render some essential identities of the Hausdorff measure of noncompactness, let us consider subsets $\mathscr{N}, \mathscr{N}_{n} \subset \mathscr{N}_{\mathbb{E}}, n \in \mathbb{N}$. Then we have:

(a) $\beta(\mathscr{N})=0$ for a relatively compact subset of $\mathbb{E}$,

(b) $\beta(\overline{\mathscr{N}})=\beta(\mathscr{N})$ where $\overline{\mathscr{N}}$ is the closure set of $\mathscr{N}$,

(c) $\beta\left(\mathscr{N}_{1}+\mathscr{N}_{2}\right) \leq \beta\left(\mathscr{N}_{1}\right)+\beta\left(\mathscr{N}_{2}\right)$,

(d) $\mathscr{N}_{1} \subset \mathscr{N}_{2}$ implies $\beta\left(\mathscr{N}_{1}\right) \leq \beta\left(\mathscr{N}_{2}\right)$,

(e) If $\left\{\mathscr{N}_{n}\right\}_{n \in \mathbb{N}}$ is a sequence of closed sets from $\mathscr{N}_{\mathbb{E}_{p}}$ such that $\mathscr{N}_{n+1} \subset \mathscr{N}_{n}$ and $\lim _{n \rightarrow \infty} \beta\left(\mathscr{N}_{n}\right)=0$, then the $\mathscr{N}_{\infty}=\bigcap_{n=1}^{\infty} \mathscr{N}_{n} \neq \phi$.

Theorem 2.1 (Darbo's theorem [33]) Let $\mathscr{N}$ be a nonempty, bounded, closed, and convex subset of a Banach space $\mathbb{E}$. Suppose that $T: \mathscr{N} \rightarrow \mathscr{N}$ is a continuous map such that there exists a constant $\varrho \in[0,1)$ with the property $\beta(T \mathscr{N}) \leq \varrho \beta(\mathscr{N})$, then $T$ has a fixed point in $\mathscr{N}$.

\subsection{Sequence spaces}

Let $\omega$ be the set of all real sequences $u=\left\{u_{i}\right\}_{i=0}^{\infty}$. A subspace $X$ of $\omega$ is said to be a $B K$ space if it is a Banach space with continuous coordinates. That is, each map $q_{i}: X \rightarrow \mathbb{C}$ defined by $q_{i}(u)=u_{i}$ is continuous for each $u \in X$ and for every $i \in \mathbb{N}$. A sequence $\left\{e_{i}\right\}_{i=0}^{\infty}$ in a Banach space $X$ is said to be a Schauder basis for $X$ if for every $u \in X$ there is a unique sequence $\left\{v_{i}\right\}_{i=0}^{\infty}$ of scalars such that $u=\sum_{i=0}^{\infty} v_{i} e_{i}$. The projection mapping $P_{n}: X \rightarrow X$ of a Banach space $X$ with a Schauder basis $\left\{e_{i}\right\}_{i=0}^{\infty}$ is given by $P_{n}(u)=\sum_{i=0}^{n} v_{i} e_{i}, u \in X$.

A $B K$ space is said to have $A K$ property if each $u \in X$ has the unique representation $u=\sum_{i=0}^{\infty} u_{i} e_{i}$. If $\|\cdot\|_{X}$ is a norm of a sequence space $\left(X,\|\cdot\|_{X}\right)$, then the norm $\|\cdot\|_{X}$ is monotone if $\left|u_{n}\right| \leq\left|v_{n}\right|$ for all $n \in \mathbb{N} \Rightarrow\|u\|_{X} \leq\|v\|_{X}$ where $u, v \in X$.

Theorem 2.2 ([1, Theorem 5.15]) Let $\mathscr{N}_{\omega}$ be a nonempty bounded subset of a BK space $\omega$ with Schauder basis $\left(e_{n}\right)$. Let $\mathscr{N} \subset \mathscr{N}_{\omega}$ and $P_{n}: \omega \rightarrow \omega, n \in \mathbb{N}$ be a projector onto the linear span of $\left\{e_{1}, e_{2}, \ldots, e_{n}\right\}$ and $I$ be the identity operator on $\omega$. Then

$$
\frac{1}{a} \lim _{n \rightarrow \infty}\left(\sup _{u \in \mathscr{N}}\left\|\left(I-P_{n}\right)(u)\right\|_{\omega}\right) \leq \beta(\mathscr{N}) \leq \lim _{n \rightarrow \infty}\left(\sup _{u \in \mathscr{N}}\left\|\left(I-P_{n}\right)(u)\right\|_{\omega}\right)
$$

where $a=\lim _{n \rightarrow \infty} \sup \left\|I-P_{n}\right\|_{\omega}$.

Remark 2.1 According to the proof of Theorem 5.16 in [1], if the space $\omega$ is a $B K$ space with $A K$ property and monotone norm $\|\cdot\|_{\omega}$, then $a=\lim _{n \rightarrow \infty} \sup \left\|I-P_{n}\right\|_{\omega}=1$.

Remark 2.2 The space of all absolutely $p$-summable sequences $\ell_{p}, 1 \leq p<\infty$ is a Banach space equipped with the norm

$$
\|u\|_{\ell_{p}}=\left(\sum_{n=0}^{\infty}\left|u_{i}\right|^{p}\right)^{\frac{1}{p}}, \quad 1 \leq p<\infty,
$$

and it is a $B K$ space with $A K$ property and monotone norm $\|\cdot\|_{\ell_{p}}$. Therefore, a nonempty bounded subset $\mathscr{N} \in \ell_{p}$ satisfies the inequality in Theorem 2.2 with $a=\lim _{n \rightarrow \infty} \sup \| I-$ $P_{n} \|_{\ell_{p}}=1$. 


\section{Basic constructions}

Consider $1 \leq p<\infty$ and let $\mathcal{C}\left([0,1], \ell_{p}\right)$ be the space of all continuous sequence functions defined on the unit interval $[0,1]$ and belonging to the space $\ell_{p}$, that is, $u(t) \in \mathcal{C}\left([0,1], \ell_{p}\right)$ implies that $u(t)=\left\{u_{i}(t)\right\}_{n=1}^{\infty} \in \ell_{p}$ and $u_{i}:[0,1] \rightarrow \mathbb{R}$ is a continuous function for all $i \in \mathbb{N}$. Define the space

$$
\mathbb{E}_{p}=\left\{u(t) \mid u \in \mathcal{C}\left([0,1], \ell_{p}\right) \text { and } \phi\left({ }_{c}^{\rho} D^{v} u(t)\right) \in \mathcal{C}\left([0,1], \ell_{p}\right), 1 \leq p<\infty\right\}
$$

equipped with the norm $\|u\|=\|u\|_{\ell_{p}}+\left\|\phi\left({ }_{c}^{\rho} D^{v} u\right)\right\|_{\ell_{p}}$ where $\phi \in \mathcal{C}\left([0,1], \ell_{p}\right)$.

Theorem 3.1 The space $\left(\mathbb{E}_{p},\|\cdot\|\right)$ is a Banach space.

Proof Let $\left\{u^{j}\right\}$ be a Cauchy sequence in the space $\left(\mathbb{E}_{p},\|\cdot\|\right)$. It is obvious that the sequences $\left\{u^{j}\right\}$ and ${ }_{c}^{\rho} D^{v}\left\{u^{j}\right\}$ are Cauchy sequences in the space $\mathcal{C}\left([0,1], \ell_{p}\right)$. Thus, the two sequences $\left\{u^{j}\right\}$ and ${ }_{c}^{\rho} D^{v}\left\{u^{j}\right\}$ are convergent and converge to $x$ and $y$, respectively, on $[0,1]$ uniformly, and $x, y \in \mathcal{C}\left([0,1], \ell_{p}\right)$. It suffices to prove that $y(t)={ }_{c}^{\rho} D^{v} x(t)$. To do this, by using (2.3), we have

$$
\begin{aligned}
\left|{ }^{\rho} I_{c}^{\nu \rho} D^{v} u^{j}(t)-{ }^{\rho} I^{v} y(t)\right| & \leq \frac{\rho^{1-v}}{\Gamma(v)} \int_{0}^{t} \frac{s^{\rho-1}}{\left(t^{\rho}-s^{\rho}\right)^{1-v}}\left|{ }_{c}^{\rho} D^{v} u^{j}(s)-y(s)\right| d s \\
& \leq \frac{1}{\rho \Gamma(v+1)} \max _{t \in[0,1]}{ }_{c}^{\rho} D^{v} u^{j}(t)-y(t) \mid .
\end{aligned}
$$

Since ${ }_{c}^{\rho} D^{v} u^{j}(t) \rightarrow y(t)$ as $j \rightarrow \infty$ uniformly on $[0,1]$, we find that ${ }^{\rho} I^{\nu}{ }_{c}^{\rho} D^{v} u^{j}(t) \rightarrow{ }^{\rho} I^{v} y(t)$ as $j \rightarrow \infty$ uniformly on $[0,1]$. Hence, by using the last relation in Lemma 2.1 , we find that $u^{j}(t)-u^{j}(0) \rightarrow{ }^{\rho} I^{v} y(t)$ as $j \rightarrow \infty$, which leads to $x(t)-c={ }^{\rho} I^{v} y(t)$ where $c$ is a constant. Applying ${ }_{c}^{\rho} D^{v}$ on both sides and using the first and third relations in Lemma 2.1, we obtain ${ }_{c}^{\rho} D^{v} x(t)=y(t)$. Hence, $\phi\left({ }_{c}^{\rho} D^{v} x(t)\right)=\phi(y(t))$.

Theorem 3.2 Let $\mathscr{N}_{\mathbb{E}_{p}}$ be a nonempty bounded subset of $\mathbb{E}_{p}, 1 \leq p<\infty$. Let $\mathscr{N} \subset \mathscr{N}_{\mathbb{E}_{p}}$, $P_{n}: \mathbb{E}_{p} \rightarrow \mathbb{E}_{p}, n \in \mathbb{N}$ be a projector and I be the identity operator on $\mathbb{E}_{p}$. Then, the Hausdorff measure of noncompactness satisfies the equality

$$
\beta(\mathscr{N})=\lim _{n \rightarrow \infty}\left(\sup _{u \in \mathscr{N}}\left\|\left(I-P_{n}\right)(u)\right\|\right) .
$$

Proof Let $u \in \mathbb{E}_{p}$, then $u \in \ell_{p}$. In view of Theorem 3.1 and the fact that $\ell_{p}, 1 \leq p<\infty$ is a $B K$ space with Schauder basis, the space $\mathbb{E}_{p}$ is a $B K$ space with the same Schauder basis. Then, according to Theorem 2.2, we get

$$
\frac{1}{a} \lim _{n \rightarrow \infty}\left(\sup _{u \in \mathscr{N}}\left\|\left(I-P_{n}\right)(u)\right\|\right) \leq \beta(\mathscr{N}) \leq \lim _{n \rightarrow \infty}\left(\sup _{u \in \mathscr{N}}\left\|\left(I-P_{n}\right)(u)\right\|\right)
$$

where $a=\lim _{n \rightarrow \infty} \sup \left\|I-P_{n}\right\|$. The left- and right-hand sides of the inequality above show that $a \geq 1$. So, it is suffices to prove that $a \leq 1$. Since $\|\cdot\|_{\ell_{p}}, 1 \leq p<\infty$ is monotone, $\left\|\left(I-P_{n}\right)(u)\right\|_{\ell_{p}} \leq\|u\|_{\ell_{p}}$ for all $u \in \ell_{p}$ and all $n \in \mathbb{N}$ (see the proof of Theorem 5.16 [1]). 
Hence, for all $n \in \mathbb{N}$, it is easy to see that

$$
\begin{aligned}
\left\|I-P_{n}\right\| & =\sup _{u \in \mathscr{N}} \frac{\left\|\left(I-P_{n}\right) u\right\|}{\|u\|} \\
& =\sup _{u \in \mathscr{N}} \frac{\left\|\left(I-P_{n}\right) u\right\|_{\ell_{p}}+\left\|\left(I-P_{n}\right) \phi\left({ }_{c}^{\rho} D^{v} u\right)\right\|_{\ell_{p}}}{\|u\|} \\
& \leq \sup _{u \in \mathscr{N}} \frac{\|u\|_{\ell_{p}}+\left\|\phi\left({ }_{c}^{\rho} D^{v} u\right)\right\|_{\ell_{p}}}{\|u\|}=\sup _{u \in \mathscr{N}} \frac{\|u\|}{\|u\|}=1 .
\end{aligned}
$$

This means that $a=\lim _{n \rightarrow \infty} \sup \left\|I-P_{n}\right\| \leq 1$, which is the desired result.

Lemma 3.1 Let $0 \leq a<b \leq c \leq 1,0<\rho \leq 1, \alpha>0$ and $p, q \geq 1$. Then,

$$
\begin{aligned}
\int_{a}^{b} s^{q(\rho-1)}\left(c^{\rho}-s^{\rho}\right)^{p(\alpha-1)} d s= & \frac{1}{\rho} c^{q(\rho-1)+\rho p(\alpha-1)+1}\left[B_{\frac{b}{c}}\left(\frac{q(\rho-1)+1}{\rho}, p(\alpha-1)+1\right)\right. \\
& \left.-B_{\frac{a}{c}}\left(\frac{q(\rho-1)+1}{\rho}, p(\alpha-1)+1\right)\right],
\end{aligned}
$$

provided that $1-1 / q<\rho \leq 1$ and $\alpha>1-1 / p$, where $B_{x}(m, n)$ is the incomplete beta function defined by

$$
B_{x}(m, n)=\int_{0}^{x} s^{m-1}(1-s)^{n-1} d s, \quad m, n>0,0<x \leq 1 .
$$

Proof Using the transform $s^{\rho}=c^{\rho} u$, we obtain the desired result.

Remark 3.1 Let $0<x \leq 1$ and $m, n>0$. Then, $0<B_{x}(m, n) \leq B(m, n)$ where $B(m, n)$ is the beta function defined by $B(m, n)=B_{1}(m, n)$.

Lemma 3.2 Let $0<\rho \leq 1,0<v<\mu \leq 1,1<\lambda \leq 2$, and $q \geq 1$. Then, the integral $\int_{0}^{1}|\mathcal{F}(t, s, \lambda, v)|^{q} d s$ is uniformly continuous for all $t \in[0,1]$ if $\rho>1-1 / q$ and the integral $\int_{0}^{1}|\mathcal{F}(t, s, 0, v)|^{q} d s$ is uniformly continuous for all $t \in[0,1]$ if $\rho(\mu-v)>1-1 / q$, where $\mathcal{F}$ is given in (2.8).

Proof Let $\alpha_{v}=\lambda+\mu-v, \beta=\mu-v+1$, and $0 \leq t_{1}<t_{2} \leq 1$. In order to prove our desired results, we notice that there are three cases: $0 \leq t_{1}<t_{2} \leq \eta<1,0 \leq t_{1} \leq \eta<t_{2} \leq 1$, and $0<\eta \leq t_{1}<t_{2} \leq 1$.

Case I. If $0 \leq t_{1}<t_{2} \leq \eta<1$, then we find that

$$
\begin{aligned}
& \frac{\Gamma^{q}\left(\alpha_{v}\right)}{\rho^{q\left(1-\alpha_{\nu}\right)}} \int_{0}^{1}\left|\mathcal{F}\left(t_{2}, s, \lambda, v\right)-\mathcal{F}\left(t_{1}, s, \lambda, v\right)\right|^{q} d s \\
& =\int_{0}^{t_{1}} s^{q(\rho-1)} \mid\left(t_{2}^{\rho}-s^{\rho}\right)^{\alpha_{\nu}-1}-\left(t_{1}^{\rho}-s^{\rho}\right)^{\alpha_{\nu}-1} \\
& \quad+\left.\frac{t_{2}^{\rho \beta}-t_{1}^{\rho \beta}}{\Omega}\left[a\left(\eta^{\rho}-s^{\rho}\right)^{\alpha_{0}-1}-\left(1-s^{\rho}\right)^{\alpha_{0}-1}\right]\right|^{q} d s \\
& \quad+\int_{t_{1}}^{t_{2}} s^{q(\rho-1)}\left|\left(t_{2}^{\rho}-s^{\rho}\right)^{\alpha_{\nu}-1}+\frac{t_{2}^{\rho \beta}-t_{1}^{\rho \beta}}{\Omega}\left[a\left(\eta^{\rho}-s^{\rho}\right)^{\alpha_{0}-1}-\left(1-s^{\rho}\right)^{\alpha_{0}-1}\right]\right|^{q} d s
\end{aligned}
$$


Salem et al. Advances in Difference Equations

(2021) 2021:132

Page 9 of 21

$$
\begin{aligned}
& +\frac{t_{2}^{q \rho \beta}-t_{1}^{q \rho \beta}}{|\Omega|^{q}}\left(\int_{t_{2}}^{\eta} s^{q(\rho-1)}\left|a\left(\eta^{\rho}-s^{\rho}\right)^{\alpha_{0}-1}-\left(1-s^{\rho}\right)^{\alpha_{0}-1}\right|^{q} d s\right. \\
& \left.+\int_{\eta}^{1} s^{q(\rho-1)}\left(1-s^{\rho}\right)^{q\left(\alpha_{0}-1\right)} d s\right) \\
\leq & 2^{q-1}\left(\int_{0}^{t_{1}} s^{q(\rho-1)}\left|\left(t_{2}^{\rho}-s^{\rho}\right)^{\alpha_{\nu}-1}-\left(t_{1}^{\rho}-s^{\rho}\right)^{\alpha_{\nu}-1}\right|^{q} d s+\int_{t_{1}}^{t_{2}} s^{q(\rho-1)}\left(t_{2}^{\rho}-s^{\rho}\right)^{q\left(\alpha_{\nu}-1\right)} d s\right) \\
& +\frac{2^{2(q-1)}\left(t_{2}^{\rho \beta}-t_{1}^{\rho \beta}\right)^{q}}{|\Omega|^{q}}\left(\int_{0}^{t_{1}} I(s) d s+\int_{t_{1}}^{t_{2}} I(s) d s\right) \\
& +\frac{t_{2}^{q \rho \beta}-t_{1}^{q \rho \beta}}{|\Omega|^{q}}\left(2^{q-1} \int_{t_{2}}^{\eta} I(s) d s+\int_{\eta}^{1} s^{q(\rho-1)}\left(1-s^{\rho}\right)^{q\left(\alpha_{0}-1\right)} d s\right) \\
= & 2^{q-1} I_{1}+\frac{2^{2(q-1)}}{|\Omega|^{q}} I_{2}+\frac{1}{|\Omega|^{q}} I_{3},
\end{aligned}
$$

where $I(s)=s^{q(\rho-1)}\left[|a|^{q}\left(\eta^{\rho}-s^{\rho}\right)^{q\left(\alpha_{0}-1\right)}+\left(1-s^{\rho}\right)^{q\left(\alpha_{0}-1\right)}\right]$. By using the well-known fact that $(x-y)^{q} \leq x^{q}-y^{q}$ for all $x \geq y>0$ and $q \geq 1$, we can deduce by Lemma 3.1, when $1<\lambda \leq 2$ $\left(\alpha_{v}>1\right)$, that

$$
\begin{aligned}
I_{1} & =\int_{0}^{t_{1}} s^{q(\rho-1)}\left|\left(t_{2}^{\rho}-s^{\rho}\right)^{\alpha_{\nu}-1}-\left(t_{1}^{\rho}-s^{\rho}\right)^{\alpha_{\nu}-1}\right|^{q} d s+\int_{t_{1}}^{t_{2}} s^{q(\rho-1)}\left(t_{2}^{\rho}-s^{\rho}\right)^{q\left(\alpha_{\nu}-1\right)} d s \\
& \leq \int_{0}^{t_{2}} s^{q(\rho-1)}\left(t_{2}^{\rho}-s^{\rho}\right)^{q\left(\alpha_{\nu}-1\right)} d s-\int_{0}^{t_{1}} s^{q(\rho-1)}\left(t_{1}^{\rho}-s^{\rho}\right)^{q\left(\alpha_{\nu}-1\right)} d s \\
& =\left(t_{2}^{q(\rho-1)+q \rho\left(\alpha_{v}-1\right)+1}-t_{1}^{q(\rho-1)+q \rho\left(\alpha_{v}-1\right)+1}\right) B\left(\frac{q(\rho-1)+1}{\rho}, q\left(\alpha_{\nu}-1\right)+1\right),
\end{aligned}
$$

and, when $\lambda=0\left(\alpha_{v}<1\right)$, we find that

$$
\begin{aligned}
I_{1} \leq & \int_{0}^{t_{1}} s^{q(\rho-1)}\left(t_{1}^{\rho}-s^{\rho}\right)^{q\left(\alpha_{v}-1\right)} d s-\int_{0}^{t_{1}} s^{q(\rho-1)}\left(t_{2}^{\rho}-s^{\rho}\right)^{q\left(\alpha_{v}-1\right)} d s \\
& +\int_{t_{1}}^{t_{2}} s^{q(\rho-1)}\left(t_{2}^{\rho}-s^{\rho}\right)^{q\left(\alpha_{\nu}-1\right)} d s \\
= & \left(t_{2}^{q(\rho-1)+q \rho\left(\alpha_{v}-1\right)+1}+t_{1}^{q(\rho-1)+q \rho\left(\alpha_{v}-1\right)+1}\right) B\left(\frac{q(\rho-1)+1}{\rho}, q\left(\alpha_{v}-1\right)+1\right) \\
- & 2 t_{2}^{q(\rho-1)+q \rho\left(\alpha_{v}-1\right)+1} B_{\left(\frac{t_{1}}{t_{2}}\right)^{\rho}\left(\frac{q(\rho-1)+1}{\rho}, q\left(\alpha_{v}-1\right)+1\right)} \\
\leq & 2 t_{2}^{q(\rho-1)+q \rho\left(\alpha_{v}-1\right)+1}\left[B\left(\frac{q(\rho-1)+1}{\rho}, q\left(\alpha_{v}-1\right)+1\right)\right. \\
& \left.-B_{\left(\frac{t_{1}}{t_{2}}\right) \rho}\left(\frac{q(\rho-1)+1}{\rho}, q\left(\alpha_{v}-1\right)+1\right)\right] .
\end{aligned}
$$

By applying the result of Lemma 3.1, we can deduce that

$$
\begin{aligned}
I_{2} & =\left(t_{2}^{\rho \beta}-t_{1}^{\rho \beta}\right)^{q}\left(|a|^{q} \int_{0}^{t_{2}} s^{q(\rho-1)}\left(\eta^{\rho}-s^{\rho}\right)^{q\left(\alpha_{0}-1\right)} d s+\int_{0}^{t_{2}} s^{q(\rho-1)}\left(1-s^{\rho}\right)^{q\left(\alpha_{0}-1\right)} d s\right) \\
& \leq\left(t_{2}^{\rho \beta}-t_{1}^{\rho \beta}\right)^{q}\left(|a|^{q} \int_{0}^{\eta} s^{q(\rho-1)}\left(\eta^{\rho}-s^{\rho}\right)^{q\left(\alpha_{0}-1\right)} d s+\int_{0}^{1} s^{q(\rho-1)}\left(1-s^{\rho}\right)^{q\left(\alpha_{0}-1\right)} d s\right)
\end{aligned}
$$




$$
=\left(t_{2}^{\rho \beta}-t_{1}^{\rho \beta}\right)^{q}\left(|a|^{q} \eta^{q(\rho-1)+q \rho\left(\alpha_{0}-1\right)+1}+1\right) B\left(\frac{q(\rho-1)+1}{\rho}, q\left(\alpha_{0}-1\right)+1\right) .
$$

Similarly, the upper bound of the integral $I_{3}$ can be evaluated as

$$
I_{3} \leq\left(t_{2}^{q \rho \beta}-t_{1}^{q \rho \beta}\right)\left[2^{q-1}\left(|a|^{q} \eta^{q(\rho-1)+q \rho\left(\alpha_{0}-1\right)+1}+1\right)+1\right] B\left(\frac{q(\rho-1)+1}{\rho}, q\left(\alpha_{0}-1\right)+1\right) .
$$

It is obvious that the integrals $I_{1}, I_{2}$, and $I_{3}$ approach uniformly zero as $t_{1} \rightarrow t_{2}$, which implies the desired results.

Case II. If $0 \leq t_{1}<\eta<t_{2} \leq 1$, then we find that

$$
\begin{aligned}
& \frac{\Gamma^{q}\left(\alpha_{\nu}\right)}{\rho^{q\left(1-\alpha_{\nu}\right)}} \int_{0}^{1}\left|\mathcal{F}\left(t_{2}, s, \lambda, v\right)-\mathcal{F}\left(t_{1}, s, \lambda, v\right)\right|^{q} d s \\
& =\int_{0}^{t_{1}} s^{q(\rho-1)} \mid\left(t_{2}^{\rho}-s^{\rho}\right)^{\alpha_{\nu}-1}-\left(t_{1}^{\rho}-s^{\rho}\right)^{\alpha_{\nu}-1} \\
& \quad+\left.\frac{t_{2}^{\rho \beta}-t_{1}^{\rho \beta}}{\Omega}\left[a\left(\eta^{\rho}-s^{\rho}\right)^{\alpha_{0}-1}-\left(1-s^{\rho}\right)^{\alpha_{0}-1}\right]\right|^{q} d s \\
& \quad+\int_{t_{1}}^{\eta} s^{q(\rho-1)}\left|\left(t_{2}^{\rho}-s^{\rho}\right)^{\alpha_{\nu}-1}+\frac{t_{2}^{\rho \beta}-t_{1}^{\rho \beta}}{\Omega}\left[a\left(\eta^{\rho}-s^{\rho}\right)^{\alpha_{0}-1}-\left(1-s^{\rho}\right)^{\alpha_{0}-1}\right]\right|^{q} d s \\
& \quad+\int_{\eta}^{t_{2}} s^{q(\rho-1)}\left|\left(t_{2}^{\rho}-s^{\rho}\right)^{\alpha_{\nu}-1}-\frac{t_{2}^{\rho \beta}-t_{1}^{\rho \beta}}{\Omega}\left(1-s^{\rho}\right)^{\alpha_{0}-1}\right|^{q} d s \\
& \quad+\frac{t_{2}^{q \rho \beta}-t_{1}^{q \rho \beta}}{|\Omega|^{q}} \int_{t_{2}}^{1} s^{q(\rho-1)}\left(1-s^{\rho}\right)^{q\left(\alpha_{0}-1\right)} d s .
\end{aligned}
$$

Case III. If $0<\eta \leq t_{1}<t_{2} \leq 1$, then we find that

$$
\begin{aligned}
& \frac{\Gamma^{q}\left(\alpha_{\nu}\right)}{\rho^{q\left(1-\alpha_{\nu}\right)}} \int_{0}^{1}\left|\mathcal{F}\left(t_{2}, s, \lambda, v\right)-\mathcal{F}\left(t_{1}, s, \lambda, v\right)\right|^{q} d s \\
& =\int_{0}^{\eta} s^{q(\rho-1)} \mid\left(t_{2}^{\rho}-s^{\rho}\right)^{\alpha_{\nu}-1}-\left(t_{1}^{\rho}-s^{\rho}\right)^{\alpha_{\nu}-1} \\
& \quad+\left.\frac{t_{2}^{\rho \beta}-t_{1}^{\rho \beta}}{\Omega}\left[a\left(\eta^{\rho}-s^{\rho}\right)^{\alpha_{0}-1}-\left(1-s^{\rho}\right)^{\alpha_{0}-1}\right]\right|^{q} d s \\
& \quad+\int_{\eta}^{t_{1}} s^{q(\rho-1)}\left|\left(t_{2}^{\rho}-s^{\rho}\right)^{\alpha_{\nu}-1}-\left(t_{1}^{\rho}-s^{\rho}\right)^{\alpha_{\nu}-1}-\frac{t_{2}^{\rho \beta}-t_{1}^{\rho \beta}}{\Omega}\left(1-s^{\rho}\right)^{\alpha_{0}-1}\right|^{q} d s \\
& \quad+\int_{t_{1}}^{t_{2}} s^{q(\rho-1)}\left|\left(t_{2}^{\rho}-s^{\rho}\right)^{\alpha_{\nu}-1}-\frac{t_{2}^{\rho \beta}-t_{1}^{\rho \beta}}{\Omega}\left(1-s^{\rho}\right)^{\alpha_{0}-1}\right|^{q} d s \\
& \quad+\frac{t_{2}^{q \rho \beta}-t_{1}^{q \rho \beta}}{|\Omega|^{q}} \int_{t_{2}}^{1} s^{q(\rho-1)}\left(1-s^{\rho}\right)^{q\left(\alpha_{0}-1\right)} d s .
\end{aligned}
$$

As in the first case, the integrals $\int_{0}^{1}|\mathcal{F}(t, s, \lambda, v)|^{q} d s$ and $\int_{0}^{1}|\mathcal{F}(t, s, 0, v)|^{q} d s$ approach uniformly zero as $t_{1} \rightarrow t_{2}$ in both cases II and III. 
Lemma 3.3 Let $\alpha_{v}=\lambda+\mu-v$ and $\beta=\mu-v+1$ where $0<\rho \leq 1,1<\lambda \leq 2$, and $0<v<$ $\mu \leq 1$. Then, for all $q \geq 1$, we have

$$
\begin{aligned}
& \sup _{t \in[0,1]} \int_{0}^{1}|\mathcal{F}(t, s, \lambda, v)|^{q} d s \\
& \quad=\frac{\rho^{1-\alpha_{\nu}}}{\Gamma\left(\alpha_{v}\right)} \min \left\{\sup _{t \in[0,1]} \int_{0}^{1} s^{q(\rho-1)}\left|\mathcal{F}_{1}(t, s, \lambda, v)\right|^{q} d s, \sup _{t \in[0,1]} \int_{0}^{1} s^{q(\rho-1)}\left|\mathcal{F}_{2}(t, s, \lambda, v)\right|^{q} d s\right\}
\end{aligned}
$$

where $\mathcal{F}_{1}, \mathcal{F}_{2}$, and $\mathcal{F}$ are given in (2.8) such that

$$
\begin{aligned}
& \sup _{t \in[0,1]} \int_{0}^{1} s^{q(\rho-1)}\left|\mathcal{F}_{1}(t, s, \lambda, \nu)\right|^{q} d s \\
& =2^{q-1} B\left(\frac{q(\rho-1)+1}{\rho}, q\left(\alpha_{v}-1\right)+1\right) \\
& \quad+\frac{2^{q-1}\left(2^{q-1}+1\right)\left(1+|a|^{q} \eta^{q(\rho-1)+q \rho\left(\alpha_{0}-1\right)+1}\right)+1}{|\Omega|^{q}} B\left(\frac{q(\rho-1)+1}{\rho}, q\left(\alpha_{0}-1\right)+1\right)
\end{aligned}
$$

and

$$
\begin{aligned}
\sup _{t \in[0,1]} & \int_{0}^{1} s^{q(\rho-1)}\left|\mathcal{F}_{2}(t, s, \lambda, v)\right|^{q} d s \\
= & 2^{q} B\left(\frac{q(\rho-1)+1}{\rho}, q\left(\alpha_{v}-1\right)+1\right) \\
& +\frac{2^{2(q-1)}\left(1+|a|^{q} \eta^{q(\rho-1)+q \rho\left(\alpha_{0}-1\right)+1}\right)+2^{q-1}+1}{|\Omega|^{q}} B\left(\frac{q(\rho-1)+1}{\rho}, q\left(\alpha_{0}-1\right)+1\right),
\end{aligned}
$$

provided that $\rho>1-1 / q$. In the case $\lambda=0$, we have to take $\rho(\mu-v)>1-1 / q$.

Proof If $0 \leq t<\eta<1$, then we find that

$$
\begin{aligned}
\int_{0}^{1} & s^{q(\rho-1)}\left|F_{1}(t, s, \lambda, \nu)\right|^{q} d s \\
= & \int_{0}^{t} s^{q(\rho-1)}\left|\left(t^{\rho}-s^{\rho}\right)^{\alpha_{\nu}-1}+\frac{t^{\rho \beta}}{\Omega}\left[a\left(\eta^{\rho}-s^{\rho}\right)^{\alpha_{0}-1}-\left(1-s^{\rho}\right)^{\alpha_{0}-1}\right]\right|^{q} d s \\
& +\frac{t^{q \rho \beta}}{|\Omega|^{q}}\left(\int_{t}^{\eta} s^{q(\rho-1)}\left|a\left(\eta^{\rho}-s^{\rho}\right)^{\alpha_{0}-1}-\left(1-s^{\rho}\right)^{\alpha_{0}-1}\right|^{q} d s\right. \\
& \left.+\int_{\eta}^{1} s^{q(\rho-1)}\left(1-s^{\rho}\right)^{q\left(\alpha_{0}-1\right)} d s\right) \\
\leq & 2^{q-1} \int_{0}^{t} s^{q(\rho-1)}\left(t^{\rho}-s^{\rho}\right)^{q\left(\alpha_{\nu}-1\right)} d s+\frac{t^{q \rho \beta}}{|\Omega|^{q}} \int_{0}^{1} s^{q(\rho-1)}\left(1-s^{\rho}\right)^{q\left(\alpha_{0}-1\right)} d s \\
& +\frac{2^{q-1}\left(2^{q-1}+1\right) t^{q \rho \beta}}{|\Omega|^{q}}\left(|a|^{q} \int_{0}^{\eta} s^{q(\rho-1)}\left(\eta^{\rho}-s^{\rho}\right)^{q\left(\alpha_{0}-1\right)}\right. \\
& \left.+\int_{0}^{1} s^{q(\rho-1)}\left(1-s^{\rho}\right)^{q\left(\alpha_{0}-1\right)} d s\right) .
\end{aligned}
$$


By Lemma 3.1, we get

$$
\begin{aligned}
\int_{0}^{1} s^{q(\rho-1)}\left|F_{1}(t, s, \lambda, \nu)\right|^{q} d s \\
\leq 2^{q-1} t^{q(\rho-1)+q \rho\left(\alpha_{\nu}-1\right)+1} B\left(\frac{q(\rho-1)+1}{\rho}, q\left(\alpha_{v}-1\right)+1\right) \\
\quad+\frac{\left(2^{2(q-1)}+2^{q-1}+1\right)+2^{q-1}\left(2^{q-1}+1\right)|a|^{q} \eta^{q(\rho-1)+q \rho\left(\alpha_{0}-1\right)+1}}{|\Omega|^{q}} \\
\quad \times t^{q \rho \beta} B\left(\frac{q(\rho-1)+1}{\rho}, q\left(\alpha_{0}-1\right)+1\right) .
\end{aligned}
$$

When $1<\lambda \leq 2$ with $\rho>1-1 / q$ or $\lambda=0$ with $\rho(\mu-v)>1-1 / q$, we have

$$
\begin{aligned}
& \int_{0}^{1} s^{q(\rho-1)}\left|\mathcal{F}_{1}(t, s, \lambda, v)\right|^{q} d s \\
& =2^{q-1} B\left(\frac{q(\rho-1)+1}{\rho}, q\left(\alpha_{v}-1\right)+1\right) \\
& \quad+\frac{2^{q-1}\left(2^{q-1}+1\right)\left(1+|a|^{q} \eta^{q(\rho-1)+q \rho\left(\alpha_{0}-1\right)+1}\right)+1}{|\Omega|^{q}} B\left(\frac{q(\rho-1)+1}{\rho}, q\left(\alpha_{0}-1\right)+1\right) .
\end{aligned}
$$

In the same way we can evaluate the upper bound of the integral $\int_{0}^{1} s^{q(\rho-1)}\left|F_{2}(t, s, \lambda, v)\right|^{q} d s$ when $0<\eta \leq t \leq 1$. The proof is finished.

\section{Main results}

By using the Hausdorff measure of noncompactness and applying Theorem 3.2 together with the Darbo's fixed point Theorem 2.1, we obtain the existence of solution for the infinite system of fractional Langevin equations (1.1) subject to the boundary conditions (1.2) with the same constraints mentioned in the first section. It is obvious, due to Lemma 2.2, that the solution of the infinite system (1.1)-(1.2) $u(t) \in \mathbb{E}_{p}$ satisfies the single integral equation

$$
u_{i}(t)=\int_{0}^{1} \mathcal{F}_{i}\left(t, s, \lambda_{i}, 0\right) h_{i}\left(s, u(s), \phi\left({ }_{c}^{\rho} D^{v} u(s)\right) d s-\xi_{i} \int_{0}^{1} \mathcal{F}_{i}(t, s, 0,0) u_{i}(s) d s\right.
$$

Here, $\mathcal{F}_{i}, i \in \mathbb{N}$ are defined as

$$
\mathcal{F}_{i}\left(t, s, \lambda_{i}, v_{i}\right)=\frac{\rho_{i}^{1+v_{i}-\lambda_{i}-\mu_{i}} s^{\rho_{i}-1}}{\Gamma\left(\lambda_{i}+\mu_{i}-v_{i}\right)} \begin{cases}\mathcal{F}_{i 1}\left(t, s, \lambda_{i}, v_{i}\right), & 0 \leq t \leq \eta_{i}<1 \\ \mathcal{F}_{i 2}\left(t, s, \lambda_{i}, v_{i}\right), & 0<\eta_{i} \leq t \leq 1\end{cases}
$$

and $\mathcal{F}_{i 1}=\mathcal{F}_{1}$ and $\mathcal{F}_{i 2}=\mathcal{F}_{2}$ where $\mathcal{F}_{1}$ and $\mathcal{F}_{2}$ are defined in (2.8) by replacing the symbols $\lambda, v, \mu, \rho, a$, and $\eta$ by the indexed symbols $\lambda_{i}, v_{i}, \mu_{i}, \rho_{i}, a_{i}$, and $\eta_{i}$, respectively. Define the sequence operators $\mathcal{P}, \mathcal{P}_{1}, \mathcal{P}_{2}: \mathbb{E}_{p} \rightarrow \mathbb{E}_{p}$ by

$$
\begin{aligned}
& \left(\mathcal{P} u_{i}\right)(t)=\left(\mathcal{P}_{1} u_{i}\right)(t)-\left(\mathcal{P}_{2} u_{i}\right)(t) \\
& \left(\mathcal{P}_{1} u_{i}\right)(t)=\int_{0}^{1} \mathcal{F}_{i}\left(t, s, \lambda_{i}, 0\right) h_{i}\left(s, u(s), \phi\left({ }_{c}^{\rho} D^{v} u(s)\right) d s,\right.
\end{aligned}
$$




$$
\left(\mathcal{P}_{2} u_{i}\right)(t)=\xi_{i} \int_{0}^{1} \mathcal{F}_{i}(t, s, 0,0) u_{i}(s) d s
$$

for all $i \in \mathbb{N}$. Their fractional derivatives of order $0<v<\mu$, by using the third identity in Lemma 2.1, can be computed as

$$
\begin{aligned}
& \left({ }_{c}^{\rho} D^{\nu_{i}} \mathcal{P}_{1} u_{i}\right)(t)=\int_{0}^{1} \mathcal{F}_{i}\left(t, s, \lambda_{i}, v_{i}\right) h_{i}\left(s, u(s), \phi\left({ }_{c}^{\rho} D^{v} u(s)\right) d s,\right. \\
& \left({ }_{c}^{\rho} D^{\nu_{i}} \mathcal{P}_{2} u_{i}\right)(t)=\xi_{i} \int_{0}^{1} \mathcal{F}_{i}\left(t, s, 0, v_{i}\right) u_{i}(s) d s .
\end{aligned}
$$

The investigation of the existence of solutions for the infinite system (1.1)-(1.2) will be discussed under the following assumptions:

$\left(\mathfrak{M}_{1}\right)$ The functions $h_{i}:[0,1] \times \ell_{p} \times \ell_{p} \rightarrow \ell_{p}$ are jointly continuous for all $i \in \mathbb{N}$ and satisfy, with Lipschitz constant $€$, the Lipschitz condition

$$
\left|h_{i}\left(t, u_{1}, v_{1}\right)-h_{i}\left(t, u_{2}, v_{2}\right)\right| \leq €\left(\left|u_{1}-u_{2}\right|+\left|v_{1}-v_{2}\right|\right), \quad u_{k}, v_{k} \in \ell_{p}, k=1,2 .
$$

$\left(\mathfrak{M}_{2}\right)$ There exist nonnegative sequence functions $x_{i}(t)$ and $y_{i}(t)$, satisfying, for all $i \in \mathbb{N}$, $t \in[0,1]$ and $u, v \in \mathcal{C}\left([0,1], \ell_{p}\right)$, the inequality

$$
\left|h_{i}(t, u, v)\right|^{p} \leq x_{i}(t)+y_{i}(t)\left(\left|u_{i}(t)\right|^{p}+\left|v_{i}(t)\right|^{p}\right)
$$

$\left(\mathfrak{M}_{3}\right)$ The sequence of functions $\left\{x_{i}(t)\right\}_{i=1}^{\infty} \in \ell_{1}$ is integrable on $[0,1]$. This means that $\lim _{n \rightarrow \infty} \sum_{i \geq n}^{\infty} x_{i}(t)=0, t \in[0,1]$, and there exists a positive constant

$$
A=\sum_{i=1}^{\infty} \int_{0}^{1} x_{i}(s) d s=\int_{0}^{1} \sum_{i=1}^{\infty} x_{i}(s) d s
$$

$\left(\mathfrak{M}_{4}\right)$ The sequence of functions $\left\{y_{i}(t)\right\}_{i=1}^{\infty}$ is equibounded on $[0,1]$. This means that there exists a positive constant $B=\sup _{t \in[0,1]} \sup _{i \in \mathbb{N}} y_{i}(t)$.

$\left(\mathfrak{M}_{5}\right)$ There exist positive constants $\mathcal{M}_{\lambda, v}$ such that

$$
\mathcal{M}_{\lambda, v}=\sup _{t \in[0,1]} \sup _{i \in \mathbb{N}}\left(\int_{0}^{1}\left|\mathcal{F}_{i}\left(t, s, \lambda_{i}, v_{i}\right)\right|^{q} d s\right), \quad q \geq 1
$$

$\left(\mathfrak{M}_{6}\right)$ The functions $\phi_{i}: \mathbb{R} \rightarrow \mathbb{R}$ are continuous and additive for all $i \in \mathbb{N}$. That is, they satisfy Cauchy's functional equation

$$
\phi_{i}(x+y)=\phi_{i}(x)+\phi_{i}(y), \quad x, y \in \mathbb{R}, i \in \mathbb{N}
$$

Remark 4.1 In view of Lemma 3.3 and Assumption $\left(\mathfrak{M}_{5}\right)$, we have

$$
\begin{aligned}
\mathcal{M}_{\lambda, v}= & \sup _{i \in \mathbb{N}} \frac{\rho_{i}^{1-\left(\lambda_{i}+\mu_{i}-v_{i}\right)}}{\Gamma\left(\lambda_{i}+\mu_{i}-v_{i}\right)}\left[\frac{2^{2(q-1)}+2^{q-1}+1+2^{2(q-1)}\left|a_{i}\right|^{q} \eta_{i}^{q\left(\rho_{i}-1\right)+q \rho_{i}\left(\lambda_{i}+\mu_{i}-1\right)+1}}{\left|\Omega_{i}\right|^{q}}\right. \\
& \times B\left(\frac{q\left(\rho_{i}-1\right)+1}{\rho_{i}}, q\left(\lambda_{i}+\mu_{i}-1\right)+1\right)
\end{aligned}
$$




$$
\begin{aligned}
& +2^{q-1} B\left(\frac{q\left(\rho_{i}-1\right)+1}{\rho_{i}}, q\left(\lambda_{i}+\mu_{i}-v_{i}-1\right)+1\right) \\
& +2^{q-1} \min \left\{B\left(\frac{q\left(\rho_{i}-1\right)+1}{\rho_{i}}, q\left(\lambda_{i}+\mu_{i}-v_{i}-1\right)+1\right),\right. \\
& \left.\left.\frac{\left|a_{i}\right|^{q} \eta_{i}^{q\left(\rho_{i}-1\right)+q \rho_{i}\left(\lambda_{i}+\mu_{i}-1\right)+1}}{\left|\Omega_{i}\right|^{q}} B\left(\frac{q\left(\rho_{i}-1\right)+1}{\rho_{i}}, q\left(\lambda_{i}+\mu_{i}-1\right)+1\right)\right\}\right] .
\end{aligned}
$$

Remark 4.2 According to Assumption $\left(\mathfrak{M}_{6}\right)$, since $\phi_{i}, i \in \mathbb{N}$ is continuous and additive, $\phi_{i}$ is a linear function and $\phi_{i}(0)=0$. Thus, there exist positive numbers $\delta_{i}$ such that $\left|\phi_{i}(x)\right|=\delta_{i}|x|$ for all $i \in \mathbb{N}$.

Theorem 4.1 Under Assumptions $\left(\mathfrak{M}_{1}\right)-\left(\mathfrak{M}_{6}\right)$ and with $\rho_{i}\left(\mu_{i}-v_{i}\right)>1 / p$ for all $i \in \mathbb{N}$ and $p>1$, the infinite system (1.1)-(1.2) has at least one solution in $\mathbb{E}_{p}$ provided that $\mathbf{S}_{\lambda}(B)+$ $\mathbf{S}_{0}\left(\xi^{p}\right)<1$ where $\mathbf{S}_{\lambda}(x)=x^{\frac{1}{p}}\left(\mathcal{M}_{\lambda, 0}^{\frac{p-1}{p}}+\delta \mathcal{M}_{\lambda, v}^{\frac{p-1}{p}}\right), \xi=\sup _{i \in \mathbb{N}}\left|\xi_{i}\right|$, and $\delta=\sup _{i \in \mathbb{N}} \delta_{i}$.

Proof In view of the continuity of the functions $h_{i}$ by Assumption $\left(\mathfrak{M}_{1}\right)$, the operator $\mathcal{P}$ defined in (4.2) is continuous. In order to prove the boundedness of the operator $\mathcal{P}$, by using Hölder's inequality for the integral and Assumption $\left(\mathfrak{M}_{5}\right)$, we obtain

$$
\begin{aligned}
\left\|\mathcal{P}_{1} u\right\|_{\ell_{p}}^{p} & \leq \sup _{t \in[0,1]} \sum_{i=1}^{\infty}\left(\int_{0}^{1}\left|\mathcal{F}_{i}\left(t, s, \lambda_{i}, 0\right)\right|\left|h_{i}\left(s, u(s), \phi\left({ }_{c}^{\rho} D^{v} u(s)\right)\right)\right| d s\right)^{p} \\
& \leq \sup _{t \in[0,1]} \sum_{i=1}^{\infty}\left(\int_{0}^{1}\left|\mathcal{F}_{i}\left(t, s, \lambda_{i}, 0\right)\right|^{q} d s\right)^{\frac{p}{q}} \int_{0}^{1}\left|h_{i}\left(s, u(s), \phi\left({ }_{c}^{\rho} D^{\mu} u(s)\right)\right)\right|^{p} d s \\
& \leq \mathcal{M}_{\lambda, 0}^{p-1} \sum_{i=1}^{\infty} \int_{0}^{1}\left[x_{i}(s)+\left.y_{i}(s)\left(\left|u_{i}(s)\right|^{p}+\mid \phi\left({ }_{c}^{\rho_{i}} D^{v_{i}} u_{i}(s)\right)\right)\right|^{p}\right] d s,
\end{aligned}
$$

where $1 / p+1 / q=1$. Invoking Assumptions $\left(\mathfrak{M}_{3}\right)$ and $\left(\mathfrak{M}_{4}\right)$,

$$
\begin{aligned}
\left\|\mathcal{P}_{1} u\right\|_{\ell_{p}} & \leq \mathcal{M}_{\lambda, 0}^{\frac{p-1}{p}}\left(A+B\left(\|u\|_{\ell_{p}}^{p}+\left\|\phi\left({ }_{c}^{\rho} D^{v} u\right)\right\|_{\ell_{p}}^{p}\right)\right)^{\frac{1}{p}} \\
& \leq \mathcal{M}_{\lambda, 0}^{\frac{p-1}{p}}\left(A^{\frac{1}{p}}+B^{\frac{1}{p}}\left(\|u\|_{\ell_{p}}+\left\|\phi\left({ }_{c}^{\rho} D^{v} u\right)\right\|_{\ell_{p}}\right)\right) \\
& =\mathcal{M}_{\lambda, 0}^{\frac{p-1}{p}}\left(A^{\frac{1}{p}}+B^{\frac{1}{p}}\|u\|\right) .
\end{aligned}
$$

Similarly, we can deduce that

$$
\left\|\mathcal{P}_{2} u\right\|_{\ell_{p}} \leq \xi \mathcal{M}_{0,0}^{\frac{p-1}{p}}\|u\|_{\ell_{p}} \leq \xi \mathcal{M}_{0,0}^{\frac{p-1}{p}}\|u\| .
$$

By using Remark 4.2, we can deduce, as above, that

$$
\begin{aligned}
& \left\|\phi\left({ }_{c}^{\rho} D^{v} \mathcal{P}_{1} u\right)\right\|_{\ell_{p}} \leq \delta \mathcal{M}_{\lambda, \nu}^{\frac{p-1}{p}}\left(A^{\frac{1}{p}}+B^{\frac{1}{p}}\|u\|\right), \\
& \left\|\phi\left({ }_{c}^{\rho} D^{\nu} \mathcal{P}_{2} u\right)\right\|_{\ell_{p}} \leq \delta \xi \mathcal{M}_{0, v}^{\frac{p-1}{p}}\|u\| .
\end{aligned}
$$


Using Minkowski inequality $\|x+y\|_{\ell_{p}} \leq\|x\|_{\ell_{p}}+\|y\|_{\ell_{p}}$ gives

$$
\begin{aligned}
\|\mathcal{P} u\| & =\left\|\mathcal{P}_{1} u-\mathcal{P}_{2} u\right\|=\left\|\mathcal{P}_{1} u-\mathcal{P}_{2} u\right\|_{\ell_{p}}+\| \phi\left({ }_{c}^{\rho} D^{v}\left(\mathcal{P}_{1} u\right)-\phi\left({ }_{c}^{\rho} D^{v}\left(\mathcal{P}_{2} u\right) \|_{\ell_{p}}\right.\right. \\
& \leq\left\|\mathcal{P}_{1} u\right\|_{\ell_{p}}+\left\|\mathcal{P}_{2} u\right\|_{\ell_{p}}+\| \phi\left({ } _ { c } ^ { \rho } D ^ { \nu } ( \mathcal { P } _ { 1 } u ) \| _ { \ell _ { p } } + \| \phi \left({ }_{c}^{\rho} D^{\nu}\left(\mathcal{P}_{2} u\right) \|_{\ell_{p}}\right.\right. \\
& \leq \mathbf{S}_{\lambda}(A)+\left[\mathbf{S}_{\lambda}(B)+\mathbf{S}_{0}\left(\xi^{p}\right)\right]\|u\| .
\end{aligned}
$$

Now, we consider the set $\mathscr{N} \subset \mathbb{E}_{p}$ given by

$$
\mathscr{N}=\left\{u \in \mathbb{E}_{p} \mid\|u\| \leq r, u \text { satisfies the boundary conditions (1.2) }\right\}
$$

which is closed, bounded, and convex, with fix $r$ satisfying the inequality $\mathbf{S}_{\lambda}(A)+\left[\mathbf{S}_{\lambda}(B)+\right.$ $\left.\mathbf{S}_{0}\left(\xi^{p}\right)\right] r \leq r$. It is clear that the operator $\mathcal{P}: \mathscr{N} \rightarrow \mathscr{N}$ is bounded. To prove the continuity of $\mathcal{P}$ on the set $\mathscr{N}$, let $u, v \in \mathscr{N}$ and assume that for all $\epsilon>0$ there exists $0<\gamma<$ $\epsilon /\left[S_{\lambda}\left(2^{p-1} \bigsqcup^{p}\right)+S_{0}\left(\xi^{p}\right)\right]$ such that $\|u-v\|<\gamma$. Then,

$$
\begin{aligned}
& \left\|\mathcal{P}_{1} u-\mathcal{P}_{1} v\right\|_{\ell_{p}} \\
& \leq \mathcal{M}_{\lambda, 0}^{\frac{p-1}{p}}\left(\sum_{i=1}^{\infty} \int_{0}^{1}\left|h_{i}\left(s, u(s), \phi\left({ }_{c}^{\rho} D^{v} u(s)\right)\right)-h_{i}\left(s, v(s), \phi\left({ }_{c}^{\rho} D^{v} v(s)\right)\right)\right|^{p} d s\right)^{\frac{1}{p}} \\
& \leq \mathcal{M}_{\lambda, 0}^{\frac{p-1}{p}}\left(\sum_{i=1}^{\infty} \mathrm{七}_{i}^{p} \int_{0}^{1}\left(|u(s)-v(s)|+\left|\phi\left({ }_{c}^{\rho} D^{v} u(s)\right)-\phi\left({ }_{c}^{\rho} D^{v} v(s)\right)\right|\right)^{p} d s\right)^{\frac{1}{p}} \\
& \leq 2^{\frac{p-1}{p}} \succeq \mathcal{M}_{\lambda, 0}^{\frac{p-1}{p}}\left(\sum_{i=1}^{\infty} \int_{0}^{1}\left(|u(s)-v(s)|^{p}+\left|\phi\left({ }_{c}^{\rho} D^{v} u(s)\right)-\phi\left({ }_{c}^{\rho} D^{v} v(s)\right)\right|^{p}\right) d s\right)^{\frac{1}{p}} \\
& \leq 2^{\frac{p-1}{p}} \succeq \mathcal{M}_{\lambda, 0}^{\frac{p-1}{p}}\left(\|u-v\|_{\ell_{p}}+\left\|\phi\left({ }_{c}^{\rho} D^{v} u\right)-\phi\left({ }_{c}^{\rho} D^{v} v\right)\right\|_{\ell_{p}}\right)=2^{\frac{p-1}{p}} \succeq \mathcal{M}_{\lambda, 0}^{\frac{p-1}{p}}\|u-v\| .
\end{aligned}
$$

By the same technique, we arrive at

$$
\|\mathcal{P} u-\mathcal{P} v\| \leq\left[S_{\lambda}\left(2^{p-1} \mathfrak{\complement}^{p}\right)+S_{0}\left(\xi^{p}\right)\right]\|u-v\|<\epsilon,
$$

which implies that the operator $\mathcal{P}$ is continuous on the set $\mathscr{N}$. To show it is continuous uniformly on the interval $[0,1]$, let $t_{0} \in[0,1]$. Then, we find that

$$
\begin{aligned}
& \left|\mathcal{P}_{1} u(t)-\mathcal{P}_{1} u\left(t_{0}\right)\right|^{p} \\
& \quad=\sum_{i=1}^{\infty}\left|\int_{0}^{1}\left[\mathcal{F}_{i}\left(t, s, \lambda_{i}, 0\right)-\mathcal{F}_{i}\left(t_{0}, s, \lambda_{i}, 0\right)\right] h_{i}\left(s, u(s), \phi\left({ }_{c}^{\rho} D^{v} u(s)\right)\right) d s\right|^{p} \\
& \quad \leq \sum_{i=1}^{\infty}\left(\int_{0}^{1}\left|\mathcal{F}_{i}\left(t, s, \lambda_{i}, 0\right)-\mathcal{F}_{i}\left(t_{0}, s, \lambda_{i}, 0\right)\right|^{q} d s\right)^{\frac{p}{q}} \int_{0}^{1}\left|h_{i}\left(s, u(s), \phi\left({ }_{c}^{\rho} D^{v} u(s)\right)\right)\right|^{p} d s,
\end{aligned}
$$

which approaches zero uniformly as $t \rightarrow t_{0}$ due to Lemma 3.2. In the same way, we can see that $\left|(\mathcal{P} u)(t)-(\mathcal{P} u)\left(t_{0}\right)\right| \rightarrow 0$ uniformly as $t \rightarrow t_{0}$, which implies that the operator $\mathcal{P}$ is continuous on $[0,1]$. 
We just have to prove that the operator $\mathcal{P}$ is condensing on the space $\mathbb{E}_{p}$ with respect to Hausdorff measure of noncompactness. Therefore, the Hausdorff measure of noncompactness $\beta(\mathscr{N})$ can be computed, according to Theorem 3.2 and Remark 2.2, as

$$
\begin{aligned}
\beta_{\mathbb{E}_{p}}(\mathscr{N} \mathcal{P}) & =\lim _{n \rightarrow \infty} \sup _{u \in \mathscr{N}}\left\|\left(I-P_{n}\right) \mathcal{P} u\right\| \\
& =\lim _{n \rightarrow \infty} \sup _{u \in \mathscr{N}}\left(\left\|\left(I-P_{n}\right) \mathcal{P} u\right\|_{\ell_{p}}+\|\left(I-P_{n}\right) \phi\left({ }_{c}^{\rho} D^{\nu} \mathcal{P} u \|_{\ell_{p}}\right)\right. \\
& =\beta_{\ell_{p}}(\mathscr{N} \mathcal{P})+\beta_{\ell_{p}}\left(\mathscr{N} \phi\left({ }_{c}^{\rho} D^{\nu} \mathcal{P}\right)\right) \\
& \leq \beta_{\ell_{p}}\left(\mathscr{N} \mathcal{P}_{1}\right)+\beta_{\ell_{p}}\left(\mathscr{N} \mathcal{P}_{2}\right)+\beta_{\ell_{p}}\left(\mathscr{N} \phi\left({ }_{c}^{\rho} D^{\nu} \mathcal{P}_{1}\right)\right)+\beta_{\ell_{p}}\left(\mathscr{N} \phi\left({ }_{c}^{\rho} D^{\nu} \mathcal{P}_{2}\right)\right) .
\end{aligned}
$$

Now, let us estimate each term separately as follows:

$$
\begin{aligned}
& \beta_{\ell_{p}}\left(\mathscr{N} \mathcal{P}_{1}\right) \\
& \quad=\lim _{n \rightarrow \infty} \sup _{u \in \mathscr{N}} \sup _{t \in[0,1]}\left(\sum_{i \geq n}^{\infty} \mid \int_{0}^{1} \mathcal{F}_{i}\left(t, s, \lambda_{i}, 0\right) h_{i}\left(s, u(s),\left.\phi\left({ }_{c}^{\rho} D^{v} u(s)\right) d s\right|^{p}\right)^{\frac{1}{p}}\right. \\
& \quad \leq \lim _{n \rightarrow \infty} \sup _{u \in \mathscr{N}} \sup _{t \in[0,1]}\left(\sum_{i \geq n}^{\infty}\left(\int_{0}^{1}\left|\mathcal{F}_{i}\left(t, s, \lambda_{i}, 0\right)\right|^{q} d s\right)^{\frac{p}{q}} \int_{0}^{1} \mid h_{i}\left(s, u(s),\left.\phi\left({ }_{c}^{\rho} D^{v} u(s)\right)\right|^{p} d s\right)^{\frac{1}{p}}\right. \\
& \leq M_{\lambda, 0}^{\frac{p-1}{p}} \lim _{n \rightarrow \infty} \sup _{u \in \mathscr{N}}\left(\sum_{i \geq n}^{\infty} \int_{0}^{1}\left[x_{i}(s)+\left.y_{i}(s)\left(\left|u_{i}(s)\right|^{p}+\mid \phi\left({ }_{c}^{\rho_{i}} D^{v_{i}} u_{i}(s)\right)\right)\right|^{p}\right] d s\right)^{\frac{1}{p}} \\
& \quad \leq M_{\lambda, 0}^{\frac{p-1}{p}} B^{\frac{1}{p}} \lim _{n \rightarrow \infty} \sup _{u \in \mathscr{N}}\left\{\left(\sum_{i \geq n}^{\infty}\left|u_{i}\right|^{p}\right)^{\frac{1}{p}}+\left(\sum_{i \geq n}^{\infty}\left|\phi\left({ }_{c}^{\rho_{i}} D^{v_{i}} u_{i}\right)\right|^{p}\right)^{\frac{1}{p}}\right\} \\
& \quad=M_{\lambda, 0}^{\frac{p-1}{p}} B^{\frac{1}{p}} \lim _{n \rightarrow \infty} \sup _{u \in \mathscr{N}}\left\{\left\|\left(I-P_{n}\right) u\right\|_{\ell_{p}}+\left\|\left(I-P_{n}\right) \phi\left({ }_{c}^{\rho} D^{v} u\right)\right\|_{\ell_{p}}\right\} \\
& \quad=M_{\lambda, 0}^{\frac{p-1}{p}} B^{\frac{1}{p}} \lim _{n \rightarrow \infty} \sup _{u \in \mathscr{N}}\left\|\left(I-P_{n}\right) u\right\|=M_{\lambda, 0}^{\frac{p-1}{p}} B^{\frac{1}{p}} \beta_{\mathbb{E}_{p}}(\mathscr{N} u) .
\end{aligned}
$$

Continuing in the same manner, we find that

$$
\beta_{\mathbb{E}_{p}}(\mathscr{N} \mathcal{P}) \leq\left[\mathbf{S}_{\lambda}(B)+\mathbf{S}_{0}\left(\xi^{p}\right)\right] \beta_{\mathbb{E}_{p}}(\mathscr{N} u)
$$

In conclusion, we checked all the assumptions of Darbo's Theorem 2.1. Therefore the infinite system (1.1)-(1.2) has at least one solution in $\mathbb{E}_{p}, p>1$.

Theorem 4.2 Under Assumptions $\left(\mathfrak{M}_{1}\right)-\left(\mathfrak{M}_{6}\right)$ and with $\rho_{i}\left(\mu_{i}-v_{i}\right)>1 / p$ for all $i \in \mathbb{N}$ and $p>1$, the infinite system (1.1)-(1.2) has at least one solution in $\mathbb{E}_{1}$ provided that $\mathbf{S}_{\lambda}(B)+$ $\mathbf{S}_{0}\left(\xi^{p}\right)<1$.

Proof It is clear that the proof of this theorem is similar to the proof of the previous one, thus it suffices to show just the last part. We have to prove that the operator $\mathcal{P}$ is condensing on the space $\mathbb{E}_{1}$ with respect to Hausdorff measure of noncompactness. 
Now, let us use the Hölder integral inequality with $1 / p+1 / q=1$ as follows:

$$
\begin{aligned}
& \beta_{\ell_{1}}\left(\mathscr{N} \mathcal{P}_{1}\right) \\
& \quad=\lim _{n \rightarrow \infty} \sup _{u \in \mathscr{N}} \sup _{t \in[0,1]} \sum_{i \geq n}^{\infty} \mid \int_{0}^{1} \mathcal{F}_{i}\left(t, s, \lambda_{i}, 0\right) h_{i}\left(s, u(s), \phi\left({ }_{c}^{\rho} D^{v} u(s)\right) d s \mid\right. \\
& \quad \leq \lim _{n \rightarrow \infty} \sup _{u \in \mathscr{N}} \sup _{t \in[0,1]} \sum_{i \geq n}^{\infty}\left(\int_{0}^{1}\left|\mathcal{F}_{i}\left(t, s, \lambda_{i}, 0\right)\right|^{q} d s\right)^{\frac{1}{q}}\left(\int_{0}^{1} \mid h_{i}\left(s, u(s),\left.\phi\left({ }_{c}^{\rho} D^{v} u(s)\right)\right|^{p} d s\right)^{\frac{1}{p}}\right. \\
& \quad \leq M_{\lambda, 0}^{\frac{1}{q}} \lim _{n \rightarrow \infty} \sup _{u \in \mathscr{N}} \sum_{i \geq n}^{\infty}\left(\int_{0}^{1}\left[x_{i}(s)+\left.y_{i}(s)\left(\left|u_{i}(s)\right|^{p}+\mid \phi\left({ }_{c}^{\rho_{i}} D^{v_{i}} u_{i}(s)\right)\right)\right|^{p}\right] d s\right)^{\frac{1}{p}} \\
& \quad \leq M_{\lambda, 0}^{\frac{p-1}{p}} B^{\frac{1}{p}} \lim _{n \rightarrow \infty} \sup _{u \in \mathscr{N}}\left\{\sum_{i \geq n}^{\infty}\left|u_{i}\right|+\sum_{i \geq n}^{\infty}\left|\phi\left({ }_{c}^{\rho_{i}} D^{v_{i}} u_{i}\right)\right|\right\}=M_{\lambda, 0}^{\frac{p-1}{p}} B^{\frac{1}{p}} \beta_{\mathbb{E}_{1}}(\mathscr{N} u) .
\end{aligned}
$$

Continuing in this manner, we find that

$$
\beta_{\mathbb{E}_{1}}(\mathscr{N} \mathcal{P}) \leq\left[\mathbf{S}_{\lambda}(B)+\mathbf{S}_{0}\left(\xi^{p}\right)\right] \beta_{\mathbb{E}_{1}}(\mathscr{N} u)
$$

Therefore the infinite system (1.1)-(1.2) has at least one solution in $\mathbb{E}_{1}$.

\section{An application}

In this section we provide an application for our main results to show how one can apply them.

Example 5.1 We introduce an infinite system of fractional Langevin equations with threepoint condition:

$$
{ }_{c}^{\frac{9}{10}} D^{\frac{3}{2}}\left({ }_{c}^{\frac{9}{10}} D^{\frac{3}{4}}+10^{-i}\right) u_{i}(t)=h_{i}\left(t, u(t), \frac{1}{5^{c}} \frac{9}{10}^{\frac{1}{8}} u(t)\right), \quad t \in[0,1], i \in \mathbb{N},
$$

subjected to the boundary conditions

$$
u_{i}(0)=0, \quad{ }_{c}^{\frac{3}{5}} D^{\frac{1}{2}} u_{i}(0)=0, \quad u_{i}(1)=5 u_{i}\left(2^{-i}\right),
$$

where $\rho=\frac{9}{10}, \lambda_{i}=\frac{3}{2}, \mu_{i}=\frac{3}{4}, v_{i}=\frac{1}{8}, \xi_{i}=10^{-i}, a_{i}=1, \eta_{i}=\frac{1}{6}$, and $\phi_{i}(x)=\frac{1}{5} x$. Also, we take

$$
h_{i}(t, u, v)=\frac{t^{2} e^{-i t} \sin (\pi t)}{(t+i)^{4} i !}+\frac{t e^{-i t}}{2(5-t)^{4}(t+i)^{2}} \sum_{n=1}^{\infty} \frac{\cos (n \pi t)}{(n+t)^{9}}\left(u_{n}(t)+v_{n}(t)\right) \text {. }
$$

It is obvious that

$$
\begin{aligned}
& \left|h_{i}(t, u, v)\right|^{p} \\
& \quad=\left|\frac{t^{4} e^{-i t} \sin (\pi t)}{(t+i)^{4} i !}+\frac{t e^{-i t}}{2(5-t)^{4}(t+i)^{2}} \sum_{n=1}^{\infty} \frac{\cos (n \pi t)}{(n+t)^{9}}\left(u_{n}(t)+v_{n}(t)\right)\right|^{p} \\
& \quad \leq 2^{p-1}\left(\left|\frac{t^{4} e^{-i t} \sin (\pi t)}{(t+i)^{4} i !}\right|^{p}+\left(\frac{t e^{-i t}}{2(5-t)^{4}(t+i)^{2}}\right)^{p}\left|\sum_{n=1}^{\infty} \frac{\cos (n \pi t)}{(n+t)^{9}}\left(u_{n}(t)+v_{n}(t)\right)\right|^{p}\right) .
\end{aligned}
$$


By using Hölder inequality, with $1 / p+1 / q=1, p, q>1$, we obtain

$$
\begin{aligned}
& \left|\sum_{n=1}^{\infty} \frac{\cos (n \pi t)}{(n+t)^{9}}\left(u_{n}(t)+v_{n}(t)\right)\right|^{p} \\
& \quad \leq\left(\sum_{n=1}^{\infty}\left|\frac{\cos (n \pi t)}{(n+t)^{9}}\left(u_{n}(t)+v_{n}(t)\right)\right|\right)^{p} \\
& \quad \leq\left(\sum_{n=1}^{\infty}\left|\frac{\cos (n \pi t)}{(n+t)^{9}}\right|^{q}\right)^{\frac{p}{q}} \sum_{n=1}^{\infty}\left|u_{n}(t)+v_{n}(t)\right|^{p} \leq\left(\sum_{n=1}^{\infty} \frac{1}{n^{9 q}}\right)^{\frac{p}{q}} \sum_{n=1}^{\infty}\left|u_{n}(t)+v_{n}(t)\right|^{p} \\
& \quad \leq 2^{p-1} \zeta^{\frac{p}{q}}(9 q) \sum_{n=1}^{\infty}\left(\left|u_{n}(t)\right|^{p}+\left|v_{n}(t)\right|^{p}\right)=2^{p-1} \zeta^{p-1}(9 q)\left(|u|^{p}+|v|^{p}\right),
\end{aligned}
$$

where $\zeta(\cdot)$ is the Riemann zeta function. When $p=1$, we have

$$
\begin{aligned}
\left|\sum_{n=1}^{\infty} \frac{\cos (n \pi t)}{(n+t)^{9}}\left(u_{n}(t)+v_{n}(t)\right)\right| & \leq \sum_{n=1}^{\infty}\left|\frac{\cos (n \pi t)}{(n+t)^{9}}\left(u_{n}(t)+v_{n}(t)\right)\right| \\
& \leq \sum_{n=1}^{\infty}\left|u_{n}(t)+v_{n}(t)\right| \leq \sum_{n=1}^{\infty}\left(\left|u_{n}(t)\right|+\left|v_{n}(t)\right|\right) \\
& =|u|+|v| .
\end{aligned}
$$

Also, we can deduce that

$$
\left|\frac{2 t^{4} e^{-i t} \sin (\pi t)}{(t+i)^{4} i !}\right|^{p} \leq \frac{2 t^{4}}{(t+1)^{4}} \frac{e^{-i t}}{i !}
$$

These estimates imply, for all $p \geq 1$, that

$$
\left|h_{i}(t, u, v)\right|^{p} \leq \frac{t^{4}}{(t+1)^{4}} \frac{e^{-i t}}{i !}+\frac{t e^{-i t}}{2(5-t)^{4} i^{2}} 2^{2(p-1)} \zeta^{p-1}(9 q)\left(|u|^{p}+|v|^{p}\right)
$$

Now, we are in a position to show that all Assumptions $\left(\mathfrak{M}_{1}\right)-\left(\mathfrak{M}_{6}\right)$ are verified for this problem:

$\left(\mathfrak{M}_{1}\right)$ Obviously, the functions $h_{i}(t, u, v)$ are continuous on $[0,1]$ for all $i \in \mathbb{N}$. In order to prove that $h_{i} \in \ell_{p}, p \geq 1$ for all $i \in \mathbb{N}$, by (5.1), we have

$$
\begin{aligned}
\|h\|^{p} & =\sum_{i=1}^{\infty}\left|h_{i}(t, u, v)\right|^{p} \\
& =\frac{t^{4}}{(t+1)^{4}} \sum_{i=1}^{\infty} \frac{e^{-i t}}{i !}+\frac{t}{2(5-t)^{4}} 2^{2(p-1)} \zeta^{p-1}(9 q)\left(|u|^{p}+|v|^{p}\right) \sum_{i=1}^{\infty} \frac{e^{-i t}}{i^{2}} \\
& =\frac{t^{4}}{(t+1)^{4}} e^{e^{-t}}+\frac{t}{2(5-t)^{4}} 2^{2(p-1)} \zeta^{p-1}(9 q)\left(|u|^{p}+|v|^{p}\right) \mathrm{L}_{2}\left(e^{-t}\right)<\infty
\end{aligned}
$$

where $\mathrm{L}_{2}$ is the dilogarithm function, which means that $h_{i} \in \ell_{p}, p \geq 1$. Also, by using (5.1), we can deduce that $h_{i}$ verify the Lipschitz condition with a positive 
constant

$$
\begin{aligned}
L & =2^{2(p-1)} \zeta^{p-1}(9 q) \sup _{t \in[0,1} \sup _{i \in \mathbb{N}} \frac{t}{2(5-t)^{4}} \frac{e^{-i t}}{i^{2}}=2^{2 p-11} \zeta^{p-1}(9 q) \\
& =\frac{43,867 \pi^{18}}{4,989,349,821,456,000} .
\end{aligned}
$$

$\left(\mathfrak{M}_{2}\right)$ We choose

$$
x_{i}(t)=\frac{t^{4}}{(t+1)^{4}} \frac{e^{-i t}}{i !} \quad \text { and } \quad y_{i}(t)=\frac{t e^{-i t}}{2(5-t)^{4} i^{2}} 2^{2(p-1)} \zeta^{p-1}(9 q)
$$

$\left(\mathfrak{M}_{3}\right)$ Since $\sum_{i=1}^{\infty} x_{i}=\frac{t^{4}}{(t+1)^{4}} e^{e^{-t}}<\infty$, we get that $x_{i} \in \ell_{1}$ and

$$
A=\sum_{i=1}^{\infty} \int_{0}^{1} x_{i}(s) d s \sim 0.0305677
$$

$\left(\mathfrak{M}_{4}\right)$ It is clear that the sequence $\left\{y_{i}(t)\right\}$ is equibounded and

$$
B=\sup _{t \in[0,1} \sup _{i \in \mathbb{N}} y_{i}(t)=2^{2 p-11} \zeta^{p-1}(9 q)=\frac{43,867 \pi^{18}}{4,989,349,821,456,000} \sim 0.00781253
$$

in the space $\mathbb{E}_{2}$, and $B=1 / 512$ in the space $\mathbb{E}_{1}$.

$\left(\mathfrak{M}_{5}\right)$ From Remark 4.1, we can evaluate the values

$$
\begin{array}{ll}
\mathcal{M}_{\frac{3}{2}, 0} \sim 3.59127, & \mathcal{M}_{\frac{3}{2}, \frac{1}{8}} \sim 3.65664 \\
\mathcal{M}_{0,0} \sim 23.1474, & \mathcal{M}_{0, \frac{1}{8}} \sim 15.5487
\end{array}
$$

in both spaces $\mathbb{E}_{2}$ and $\mathbb{E}_{1}$.

$\left(\mathfrak{M}_{6}\right)$ According to Remark 4.2, $\delta_{i}=5^{-i}$ for all $i \in \mathbb{N}$ and $\delta=\max _{i \in \mathbb{N}} \delta_{i}=\frac{1}{5}$.

Therefore, all Assumptions $\left(\mathfrak{M}_{1}\right)-\left(\mathfrak{M}_{6}\right)$ are verified,

$$
\rho_{i}\left(\mu_{i}-v_{i}\right)=\frac{9}{10}\left(\frac{3}{4}-\frac{1}{8}\right)=\frac{9}{16}>\frac{1}{2}=\frac{1}{p}
$$

in both spaces $\mathbb{E}_{2}$ and $\mathbb{E}_{1}$, and

$$
\begin{array}{ll}
\mathbf{S}_{\lambda}(B)+\mathbf{S}_{0}\left(\xi^{p}\right) \sim 0.761287<1 & \text { in } \mathbb{E}_{2}, \\
\mathbf{S}_{\lambda}(B)+\mathbf{S}_{0}\left(\xi^{p}\right) \sim 0.660634<1 & \text { in } \mathbb{E}_{1},
\end{array}
$$

which implies that our example verifies the conditions of Theorems 4.1 and 4.2. Thus, the problem of Example 5.1 has at least one solution in the space $\mathbb{E}_{2}$ and also in $\mathbb{E}_{1}$.

\section{Conclusion}

In the present research, we studied an infinite system of Langevin equations of fractional order. The fractional derivative used in our model is the so-called generalized LiouvilleCaputo derivative, which associates with many well-known fractional derivatives. By applying the measure of noncompactness technique and using the Darbo's fixed point theorem, we examined the existence of solution to this infinite system. This investigation has 
been performed in a new sequence space related to the $\ell_{p}, 1 \leq p<\infty$ space. A numerical example is presented to illustrate our idea by investigating a function satisfying all the proposed assumptions.

\section{Acknowledgements}

We are very grateful to the anonymous referees for their suggestions and valuable advices.

\section{Funding}

This research work was funded by Institutional Fund Project under grant No. (IFPHI-339-130-2020). Therefore, authors gratefully acknowledge the technical and financial support from the Ministry of Education and King Abdulaziz University, DSR, Jeddah, Saudi Arabia.

\section{Availability of data and materials}

Not applicable.

\section{Competing interests}

The authors declare that they have no competing interests.

\section{Authors' contributions}

All authors contributed equally and significantly in writing this paper. All authors read and approved the final manuscript.

\section{Author details}

'Department of Mathematics, Faculty of Science, King Abdulaziz University, P.O. Box 80203, Jeddah, 21589, Saudi Arabia.

${ }^{2}$ Department of Mathematics, University of Jeddah, Jeddah, 41510, Saudi Arabia.

\section{Publisher's Note}

Springer Nature remains neutral with regard to jurisdictional claims in published maps and institutional affiliations.

Received: 2 November 2020 Accepted: 14 February 2021 Published online: 25 February 2021

\section{References}

1. Banas, J., Mursaleen, M.: Sequence Spaces and Measures of Noncompactness with Applications to Differential and Integral Equations. Springer, Delhi (2014)

2. Deimling, K.: Ordinary Differential Equations in Banach Spaces. Lect. Notes Math., vol. 596. Springer, Berlin (1977)

3. Banas, J., Lecko, M.: Solvability of infinite systems of differential equations in Banach sequence spaces. J. Comput. Appl. Math. 137, 363-375 (2001)

4. Mursaleen, M., Mohiuddine, S.A.: Applications of measures of noncompactness to the infinite system of differential equations in $\ell_{p}$ spaces. Nonlinear Anal. 75, 2111-2115 (2012)

5. Mursaleen, M.: Application of measure of noncompactness to infinite systems of differential equations. Can. Math. Bull. 56(2), 388-394 (2013)

6. Mursaleen, M., Rizvi, S.M.H.: Solvability of infinite system of second order differential equations in $c_{0}$ and $c_{1}$ by Meir-Keeler condensing operator. Proc. Am. Math. Soc. 144(10), 4279-4289 (2016)

7. Banas, J., Mursaleen, M., Rizvi, S.M.H.: Existence of solutions of a boundary value problem for an infinite system of differential equations. Electron. J. Differ. Equ. 2017, 262 (2017)

8. Mursaleen, M., Bilalov, B., Rizvi, S.M.H.: Applications of measures of noncompactness to infinite system of fractional differential equations. Filomat 31(11), 3421-3432 (2017)

9. Seemab, A., Ur Rehman, M.: Existence of solution of an infinite system of generalized fractional differential equations by Darbo's fixed point theorem. J. Comput. Appl. Math. 364, 112355 (2020)

10. Kilbas, A.A., Srivastava, H.M., Trujillo, J.J.: Theory and Applications of Fractional Differential Equations. North-Holland Mathematics Studies. Elsevier, Amsterdam (2006)

11. Srivastava, H.M.: Fractional-order derivatives and integrals: introductory overview and recent developments. Kyungpook Math. J. 60, 73-116 (2020). https://doi.org/10.5666/KMJ.2020.60.1.73

12. Srivastava, H.M.: Diabetes and its resulting complications: mathematical modeling via fractional calculus. Publ. Health 4(3), ID: 2, 1-15 (2020). https://doi.org/10.23880/phoa-16000163

13. Katugampola, U.N.: Existence and uniqueness results for a class of generalized fractional differential equations (2016). arXiv:1411.5229v2 [math.CA]

14. Coffey, W.T., Kalmykov, Y.P., Waldron, J.T.: The Langevin Equation, 2nd edn. World Scientific, Singapore (2004)

15. Ahmad, B., Alghanmi, M., Alsaedi, A., Srivastava, H.M., Ntouyas, S.N.: The Langevin equation in terms of generalized Liouville-Caputo derivatives with nonlocal boundary conditions involving a generalized fractional integral. Mathematics 7, 533 (2019)

16. Salem, A.: Existence results of solutions for ant-periodic fractional Langevin equation. J. Appl. Anal. Comput. 10(6) 2557-2574 (2020). https://doi.org/10.11948/20190419

17. Salem, A., Mshary, N.: On the existence and uniqueness of solution to fractional-order Langevin equation. Adv. Math. Phys. 2020, Article ID 8890575 (2020). https://doi.org/10.1155/2020/8890575

18. Lim, S.C., Li, M., Teo, L.P.: Langevin equation with two fractional orders. Phys. Lett. A 372, 6309-6320 (2008)

19. Lozinski, A., Owen, R.G., Philips, T.N.: The Langevin and Fokker-Planck equations in polymer rheology. In: Handbook of Numerical Analysis, vol. 16, pp. 211-303 (2011)

20. Salem, A., Alzahrani, F., Almaghamsi, L.: Fractional Langevin equation with nonlocal integral boundary condition. Mathematics 7(5), 402 (2019) 
21. Salem, A., Alghamdi, B.: Multi-point and anti-periodic conditions for generalized Langevin equation with two fractional orders. Fractal Fract. 3(4), 51 (2019)

22. Salem, A., Alzahrani, F., Alnegga, M.: Coupled system of non-linear fractional Langevin equations with multi-point and nonlocal integral boundary conditions. Math. Probl. Eng. 2020, Article ID 7345658 (2020)

23. Salem, A., Alzahrani, F., Alghamdi, B.: Langevin equation involving two fractional orders with three-point boundary conditions. Differ. Integral Equ. 33, 163-180 (2020)

24. Salem, A., Alnegga, M.: Measure of noncompactness for hybrid Langevin fractional differential equations. Axioms $9(2), 59(2020)$

25. Salem, A., Alzahrani, F., Al-Dosari, A.: Attainability to solve fractional differential inclusion on the half line at resonance. Complexity 2020, Article ID 9609108 (2020). https://doi.org/10.1155/2020/9609108

26. Salem, A., Al-Dosari, A.: Existence results of solution for fractional Sturm-Liouville inclusion involving composition with multi-maps. J. Taibah Univ. Sci. 14(1), $721-733$ (2020)

27. Fazli, H., Nieto, J.J.: Fractional Langevin equation with anti-periodic boundary conditions. Chaos Solitons Fractals 114, 332-337 (2018)

28. Baghani, H.: An analytical improvement of a study of nonlinear Langevin equation involving two fractional orders in different intervals. J. Fixed Point Theory Appl. 21, 95 (2019)

29. Baghani, H., Nieto, J.J.: On fractional Langevin equation involving two fractional orders in different intervals. Nonlinear Anal., Model. Control 24, 884-897 (2019)

30. Katugampola, U.N.: New approach to a generalized fractional integral. Appl. Math. Comput. 218, 860-865 (2011)

31. Kiryakova, V.: Generalized Fractional Calculus and Applications. Longman, Harlow (1994)

32. Jarad, F., Abdeljawad, T., Baleanu, D.: On the generalized fractional derivatives and their Caputo modification. J. Nonlinear Sci. Appl. 10, 2607-2619 (2017)

33. Banas, J., Goebel, K.: Measures of Noncompactness in Banach Spaces. Lecture Notes in Pure and Applied Mathematics, vol. 60. Dekker, New York (1980)

\section{Submit your manuscript to a SpringerOpen ${ }^{\circ}$ journal and benefit from:}

- Convenient online submission

- Rigorous peer review

- Open access: articles freely available online

- High visibility within the field

- Retaining the copyright to your article

Submit your next manuscript at $\boldsymbol{\nabla}$ springeropen.com 